\title{
Bioinformatics and Machine Learning Methods to Identify FN1 as a Novel Biomarker of Aortic Valve Calcification
}

\author{
Tao Xiong ${ }^{1 \dagger}$, Shen Han ${ }^{1,2 \dagger}$, Lei Pu ${ }^{1,2}$, Tian-Chen Zhang ${ }^{1}$, Xu Zhan ${ }^{3}$, Tao Fu ${ }^{1}$, Ying-Hai Dai ${ }^{1}$ \\ and Ya-Xiong $\mathrm{Li}^{1,2 *}$ \\ ${ }^{1}$ Cardiovascular Surgery, Yan'an Affiliated Hospital of Kunming Medical University, Kunming Medical University, Kunming, \\ China, ${ }^{2}$ Key Laboratory of Cardiovascular Disease of Yunnan Province, Yan'an Affiliated Hospital of Kunming Medical \\ University, Kunming Medical University, Kunming, China, ${ }^{3}$ Department of Thoracic and Cardiovascular Surgery, The First \\ Affiliated Hospital of Chongqing Medical University, Chongqing, China
}

\section{OPEN ACCESS}

Edited by:

Sasha A. Singh,

Brigham and Women's Hospital and Harvard Medical School, United States

Reviewed by:

Alexander Akhmedov, University of Zurich, Switzerland Jun-ichiro Koga,

Kyushu University, Japan

*Correspondence: Ya-Xiong L liyaxiong62@aliyun.com; lyxyayy@163.com

tThese authors have contributed equally to this work

Specialty section: This article was submitted to Atherosclerosis and Vascular Medicine

a section of the journal Frontiers in Cardiovascular Medicine

Received: 10 December 2021 Accepted: 28 January 2022 Published: 28 February 2022

Citation:

Xiong T, Han S, Pu L, Zhang T-C, Zhan X, Fu T, Dai Y-H and Li Y-X (2022) Bioinformatics and Machine Learning Methods to Identify FN1 as a Novel Biomarker of Aortic Valve Calcification.

Front. Cardiovasc. Med. 9:832591. doi: 10.3389/fcvm.2022.832591
Aim: The purpose of this study was to identify potential diagnostic markers for aortic valve calcification (AVC) and to investigate the function of immune cell infiltration in this disease.

Methods: The AVC data sets were obtained from the Gene Expression Omnibus. The identification of differentially expressed genes (DEGs) and the performance of functional correlation analysis were carried out using the $\mathrm{R}$ software. To explore hub genes related to AVC, a protein-protein interaction network was created. Diagnostic markers for AVC were then screened and verified using the least absolute shrinkage and selection operator, logistic regression, support vector machine-recursive feature elimination algorithms, and hub genes. The infiltration of immune cells into AVC tissues was evaluated using CIBERSORT, and the correlation between diagnostic markers and infiltrating immune cells was analyzed. Finally, the Connectivity Map database was used to forecast the candidate small molecule drugs that might be used as prospective medications to treat AVC.

Results: A total of 337 DEGs were screened. The DEGs that were discovered were mostly related with atherosclerosis and arteriosclerotic cardiovascular disease, according to the analyses. Gene sets involved in the chemokine signaling pathway and cytokine-cytokine receptor interaction were differently active in AVC compared with control. As the diagnostic marker for AVC, fibronectin 1 (FN1) (area the curve $=0.958$ ) was discovered. Immune cell infiltration analysis revealed that the AVC process may be mediated by naïve B cells, memory B cells, plasma cells, activated natural killer cells, monocytes, and macrophages M0. Additionally, FN1 expression was associated with memory B cells, MO macrophages, activated mast cells, resting mast cells, monocytes, and activated natural killer cells. AVC may be reversed with the use of yohimbic acid, the most promising small molecule discovered so far.

Conclusion: FN1 can be used as a diagnostic marker for AVC. It has been shown that immune cell infiltration is important in the onset and progression of AVC, which may benefit in the improvement of AVC diagnosis and treatment.

Keywords: aortic valve calcification, immune infiltration, diagnostic, biomarker, CIBERSORT, small molecule drugs 


\section{INTRODUCTION}

Aortic valve calcification (AVC) is the most frequent kind of valvular disease in the world, and is the most common cause of aortic stenosis $(1,2)$. There are between 2 and $7 \%$ of the population aged 65 and older who are affected by this disease (3). As the condition worsens over time, more and more fibrous calcification and pathological thickening of the aortic valve lead to a reduction in the mobility of the aortic valve leaflets, and ultimately lead to serious obstruction of the heart outflow (4). The disease burden of AVC is expected to double over the next 50 years, posing a serious threat to public health in an aging world (4). Although the therapies used to treat aortic valve stenosis have made progress, including valve replacement and interventional therapy, the exact biological process of AVC is still mystery.

After statins failed to slow the progression of AVC, angiotensin-converting enzyme inhibitors also failed, and there is still no progress in the effectiveness of drug treatments (5-8). To date, early surgery is the only effective treatment option for improving clinical outcomes in patients with AVC (9). However, surgery is related to high expenses, an inevitability of mortality, as well as perioperative and long-term morbidity, such as those related with anticoagulant medication and the necessity for reoperation due to prosthetic valve failure. As a result, an urgent medical need was recognized for a comprehensive understanding of fundamental processes of AVC as well as for the development of new treatment targets to slow the advancement of the disease's evolution. In order to diagnose and cure early, biomarkers and pathways should be identified. This is essential for early recognition, prevention, and accurate therapy.

Histopathological analysis reveals that the beginning phase of AVC is an aggressive inflammation reaction, similar to that of atherosclerotic lesions, consisting of processes ranging from lipid deposition, infiltration of inflammatory cells such as macrophages and $\mathrm{T}$ cells, and finally leading to the destruction of the basement membrane (10). The final stage of AVC is typically characterized by heterotopic ossification, which includes mature lamellar bone formation and active bone remodeling (11). According to several studies, signal transduction channels that are associated with the progression of aortic calcification are composed of various growth factors, cytokines, and tumor necrosis factors (12-14). Current knowledge of the mechanisms underlying AVC progression, including molecular actions, cellular functions and biomechanics, has been established. The most important of these are the morphology of the mitral aortic valve, disturbances in endocrine regulation, valve osteogenesis,

\footnotetext{
Abbreviations: AVC, aortic valve calcification; DEGs, differentially expressed genes; PPIN, protein-protein interaction network; FN1, fibronectin 1; GO, Gene Ontology; DO, Disease Ontology; GSEA, Gene set enrichment analysis; STRING, The Search Tool for the Retrieval of Interacting Genes; PPI, protein-protein interaction; LASSO, least absolute shrinkage and selection operator; SVM-RFE, support vector machine-recursive feature elimination; KEGG, Kyoto Encyclopedia of Genes and Genomes; CXCL8, C-X-C motif chemokine ligand 8; CXCR4, C-XC motif chemokine receptor 4; CCR5, C-C motif chemokine receptor 5; MMP9, matrix metallopeptidase 9; SYK, spleen-associated tyrosine kinase; TYROBP, transmembrane immune signaling adaptor; CXCL12, C-X-C motif chemokine ligand 12; SDC1, syndecan 1; TLR2, toll-like receptor 2; IL, interleukin; NF, necrosis factor.
}

dysregulation of mineral metabolism, and lack of signaling by osteoclasts (15).

In this study, we used CIBERSORT for the first time to analyze the expression matrix of AVC and normal tissue samples, and calculated the proportions of their immune cells. Moreover, we investigated the association between the biomarkers discovered and the infiltrating immune cells, providing the groundwork for future study in this area. Most importantly, we have well screened potential AVC targeted small molecule drugs, by using the connection map (CMap) database.

\section{MATERIALS AND METHODS \\ Data Download and Processing}

The GSE12644 (16) and GSE51472 (17) datasets were obtained from http://www.ncbi.nlm.nih.gov/geo/ (18), which the GPL570 platform of the 84 Affymetrix Human Genome U133 Plus 2.0 Aray served as the foundation. The GSE12644 dataset contained $10 \mathrm{AVC}$ and 10 controls that were collected from the aortic valve, and the GSE51472 dataset contained 10 AVC and 5 controls that were collected from the aortic valve. The probes in each dataset were translated to gene symbols based on the probe annotation files that were provided by the researchers. As several probes correspond to the same gene symbol, we use the average value of the probes to represent the level of expression of that gene in the tissue. In order to conduct additional integration research, the two datasets were pooled into a metadata cohort. This was done since GSE12644 and GSE51472 both share a similar platform and are beneficial for merging data. Before performing normalization, the expression values from both datasets were $\log 2$ transformed. ComBat outperforms other tools in a systematic evaluation. As a result, we choose ComBat in order to eliminate the batch effect between the two datasets (19). We performed principal component analysis (PCA) to determine if the batch effect had been eliminated. Additionally, we identified an aortic valve disease data set (GSE83453) (20) from the GEO database to serve as a validation cohort, consisting of nine AVC samples and eight control samples, which was performed using the Illumina HumanHT-12 v4.0 Gene Expression BeadChip (Laval University, Quebec, Canada; anthor:Yohan Bossé).

\section{Identification of DEGs}

The "limma" package (21) was used to screen for DEGs, and heat and volcano maps of DEGs were created by the "ggplot2" package (22) to visualize their differential expression. With the $\mathrm{P}$ value $<0.05$ and $|\log 2 \mathrm{FC}|>0.585$, DEGs were deemed statistically significant in this study.

\section{Functional Correlation Analysis}

We performed Gene Ontology (GO), Kyoto Encyclopedia of Genes and Genomes (KEGG) and Disease Ontology (DO) enrichment analyses of DEGs using the "clusterProfiler" package (23). Gene set enrichment analysis (GSEA) of the gene expression matrix was performed using the "clusterProfiler" package, with the reference gene sets "c5.go.v7.4.symbols.gmt" and "c2.cp.kegg.v7.0.symbols.gmt" being used. $q$-value $<0.05$ and adjusted $P$-value $<0.05$ were considered significantly enriched. 


\section{Hub Genes Screening}

The Search Tool for the Retrieval of Interacting Genes (STRING) (24) database was used to analyze functional protein association networks. The screened DEGs were submitted to the STRING database. All protein-protein interaction (PPI) pairs with a combined score of $>0.7$ were extracted. To maintain the overall network's stability, high-degree nodes seemed to be essential. We use Cytoscape (v3.6.1) plug-in cytoHubba to calculate the degree of all nodes (25). In this study, the top 10 genes with the greatest degree rank are identified key genes.

\section{Verification of Diagnostic Markers}

To screen diagnostic markers for AVC, the least absolute shrinkage and selection operator (LASSO) logistic regression (26) as well as support vector machine-recursive feature elimination (SVM-RFE) (27) were used by us. After quality control, the expression matrices of the GSE12644 and GSE51472 datasets were combined to create an independent dataset, and the combined diagnosing efficacy of the gathered diagnostic markers was determined using this dataset. The LASSO algorithm was used in conjunction with the "glmnet" package (28). SVM-RFE is a support vector machine-based machine learning technique, which is used to exclude SVM-generated eigenvectors in order to select the optimal variables. The "e1071" package established an SVM module to further characterize the diagnostic utility of the biomarkers in AVC (29).We integrated the hub genes with the PPI network, LASSO and SVM-RFE algorithms to conduct a more in-depth study. $P<0.05$ was regarded statistically significant on a two-sided basis.

\section{Evaluation of Immune Cell Infiltration}

In CIBERSORT (https://cibersortx.stanford.edu/), we filtered out samples with a $P<0.05$ and acquired the matrix about immune cell infiltration as a result of submitting the gene expression matrix information. The "ggplot2" software was then used to conduct PCA analysis on the immune cell infiltration matrix input, resulting in the creation of a two-dimensional PCA map. The "corrplot" software is responsible for plotting correlative data. Correlation heatmaps were created using the "corrplot" package (30) in order to show the relationship between 22 distinct kinds of infiltration immune cells. It was necessary to build violin diagrams to display the discrepancies in immune cell infiltration, which was accomplished using the "ggplot2" package.

\section{Immune Cells and Diagnostic Markers}

The relationship between the amounts of infiltrating immune cells and the levels of the discovered gene biomarkers was investigated using Spearman's rank correlation test in the $\mathrm{R}$ software. The chart method provided by the "ggplot2" package was used to display the generated correlations.

\section{Identification of Small Molecules}

CMap (http://www.broadinstitute.org/cmap/) is a repository of databases containing thousands of gene transcription profiles, obtained from cultured mammalian cells exposed to active small molecule drugs. It was searched to identify small molecule therapeutic candidates with the AVC gene signature. DEGs were classified into two groups: up-regulated and down-regulated groups. The similarity was quantified using enrichment scores ranging from -1 to +1 . A positive connecting value (near to +1 ) suggests that a small compound may trigger the expression of the AVC gene, while a negative connecting value (near to -1 ) implies that a compound can mimic the condition of normal cells.

\section{RESULTS}

Supplementary File 1 lists data matrix information of the training datasets (GSE12644 and GSE51472) and validation dataset (GSE83453).

\section{Data Preprocessing and DEG Screening}

The batch effect between GSE12644 and GSE51472 was evaluated and visualized using a PCA cluster diagram. A batch effect existed between them (Figure 1A), and the interbatch variation had been eliminated. After normalization and processing, two two-dimensional PCA cluster diagrams were used to display the combined gene expression matrix before normalization (Supplementary File 2) and after normalization (Supplementary File 3), respectively (Figure 1B). After normalization, the clustering of the two sample groups was more evident, indicating a reliable sample source. Following data preparing, we used $\mathrm{R}$ to identify 337 DEGs from the normalized data, as described by the heat map as well as volcano map (Figures 2A,B). Compared with normal samples, 203 of these DEGs were upregulated and 134 were downregulated in AVC. The top 10 upregulated and downregulated genes are summarized in Table 1.

\section{Functional Correlation Analysis}

The findings of the GO analysis were separated into three subcategories, which were biological process, cell component, and molecular function, respectively (Figure 3A, Supplementary File 4). Cell chemotaxis, leukocyte chemotaxis, extracellular matrix organization, extracellular structure organization, external encapsulating structure organization, neutrophil activation, neutrophil degranulation, neutrophil activation involved in immune response, neutrophil-mediated immunity, and myeloid leukocyte migration were all observed during the biological process. The DEGs were enriched in cell components including the extracellular matrix containing collagen, the external side of the plasma membrane, collagen trimer, complex of collagen trimers, secretory granule lumen, cytoplasmic vesicle lumen, vesicle lumen, endoplasmic reticulum lumen, tertiary granule, and ficolin-1-rich granule. The DEGs were enriched in chemokine activity, extracellular matrix structural constituent, cytokine activity, chemokine receptor binding, and integrin binding for molecular function. Otherwise, the KEGG pathway was enriched for viral protein interaction with cytokine and cytokine receptor, chemokine signaling pathway, extracellular matrix-receptor interaction, rheumatoid arthritis, and cytokine-cytokine receptor interaction (Figure 3B, Supplementary File 4). The findings of the DO study are depicted in Figure 4 and Supplementary File 4. The most common diseases enhanced by DEGs were osteoarthritis, lung 
A

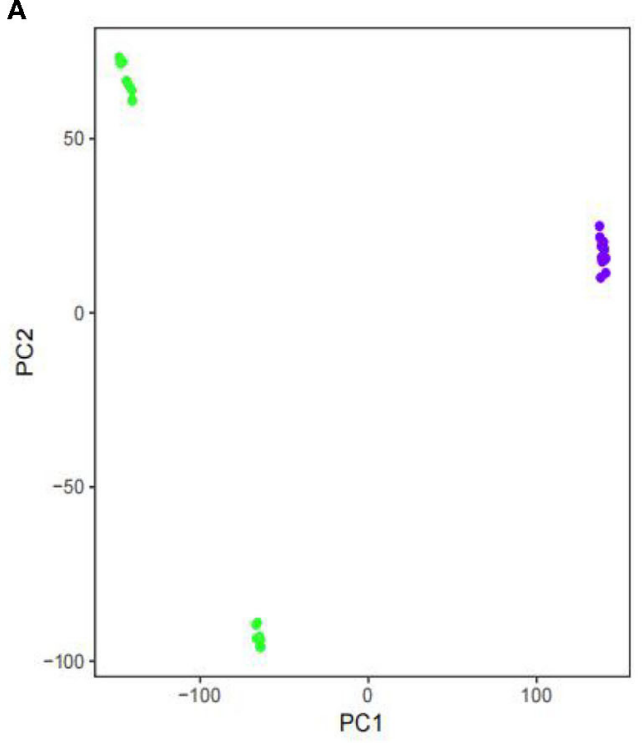

B

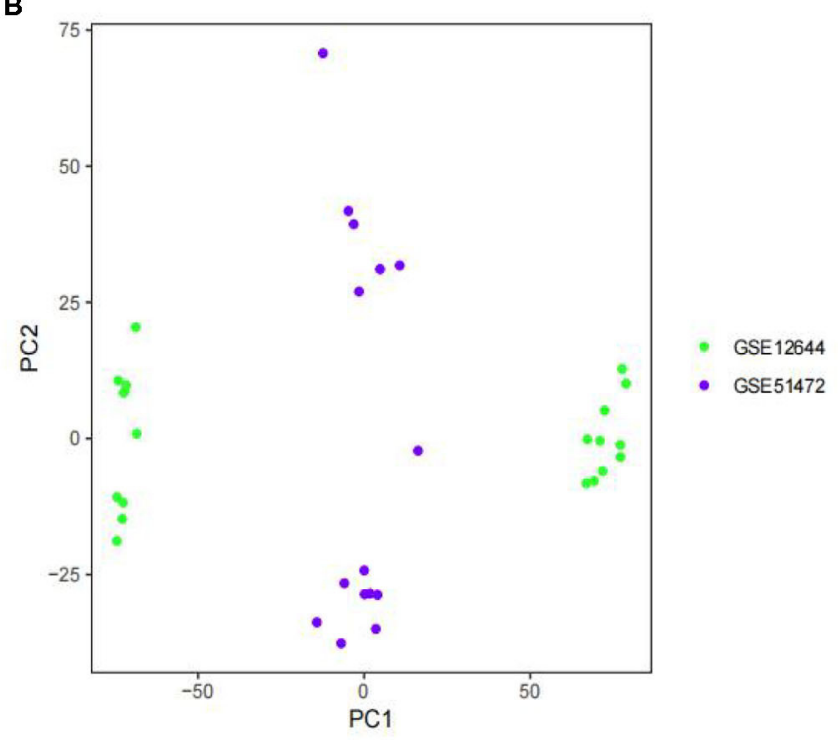

FIGURE 1 | Principal component analysis (PCA) analysis of gene expression datasets. The scatter plots' points depict samples based on the top two principal components (PC1 and PC2) of gene expression profiles without and with batch effect removal. (A) PCA cluster plot of GSE12644 and GSE51472 before sample correction and remove batch effect. (B) PCA cluster plot of GSE12644 and GSE51472 after sample correction and remove batch effect. The colors denote samples from two distinct datasets, respectively. Each dot represents a sample; green represents a sample from GSE12644; purple represents a sample from GSE51472.

disease, chronic obstructive pulmonary disease, obstructive lung disease, arteriosclerosis, arteriosclerotic cardiovascular disease, atherosclerosis, myocardial infarction, and coronary artery disease. GSEA results indicated that in normal samples, GO biological processes primarily involved fatty acid beta oxidation, mRNA processing, RNA splicing, and RNA splicing via transesterification reaction (Figure 5A, Supplementary File 5). In disease samples, GO biological processes primarily involved inactivation of immune response and adaptive immune response based on somatic recombination of immune receptors built (Figure 5B, Supplementary File 5). The KEGG enrichment analysis in the normal group (Figure 6A, Supplementary File 5) revealed significant enrichment in fatty acid metabolism, histidine metabolism, xenobiotic metabolism via cytochrome $\mathrm{p} 4$, propanoate metabolism, and valine leucine and isoleucine degradation. In the disease group, significantly enriched in the KEGG pathway (Figure 6B, Supplementary File 5) including chemokine signaling pathway, cytokine-cytokine receptor interaction, and hematopoietic cells. The aforementioned results indicate that that the immune response significantly affects the process of AVC.

\section{Hub Genes Screening}

STRING tools were used to predict the protein-protein interactions of the 337 DEGs. The protein-protein interaction network contained 165 nodes and 420 edges (Figure 7A). The top 10 key genes are identified by the degree of connectivity in the protein-protein interaction network. With a connection degree of 28, fibronectin 1 (FN1) was the most prominent gene, followed by C-X-C motif chemokine ligand 8 (CXCL8) (degree
$=23$ ), $\mathrm{C}-\mathrm{X}-\mathrm{C}$ motif chemokine receptor 4 (CXCR4) (degree $=23$ ), $\mathrm{C}-\mathrm{C}$ motif chemokine receptor 5 (CCR5) (degree $=$ 20), matrix metallopeptidase 9 (MMP9) (degree =19), spleenassociated tyrosine kinase $(\mathrm{SYK})($ degree $=19)$, transmembrane immune signaling adaptor TYROBP (TYROBP) (degree $=18)$, $\mathrm{C}-\mathrm{X}-\mathrm{C}$ motif chemokine ligand $12(\mathrm{CXCL} 12)($ degree $=18)$, syndecan $1(\mathrm{SDC} 1)$ (degree $=18)$, and toll-like receptor 2 (TLR2) (degree $=17$ ). All of the key genes listed above are upregulated. Additionally, the PPIN of the 10 discovered hub genes were created, indicating a substantial interaction between them (Figure 7B).

\section{Verification of Diagnostic Markers}

To use the LASSO logistic regression approach, we were able to recognize 11 genes from DEGs (Figure 8A). The SVMRFE technique is used to determine eight genes from DEGs (Figure 8B). By combining the genes identified by the two techniques and the hub gene, a diagnostic-related gene was generated (Figure 8C). FN1 was matched to a variable and had a diagnostic efficiency of 0.958 in the training set (Figure 8D). To further evaluate the diagnostic efficacy of FN1, we used the GSE83453 dataset as the validation set (Figure 8E). FN1 achieved a high level (area the curve $=0.833$ ) (Figure 8F), showing that FN1 had a high diagnostic value.

\section{Results of Immune Cell Infiltration}

GSE12644 and GSE51472 merged data matrices were analyzed using CIBERSORT and the results are described in Supplementary File 6. In order to examine the uniformity of biological repetition and the variation between the AVC and the normal samples, PCA analysis is used. On the basis 


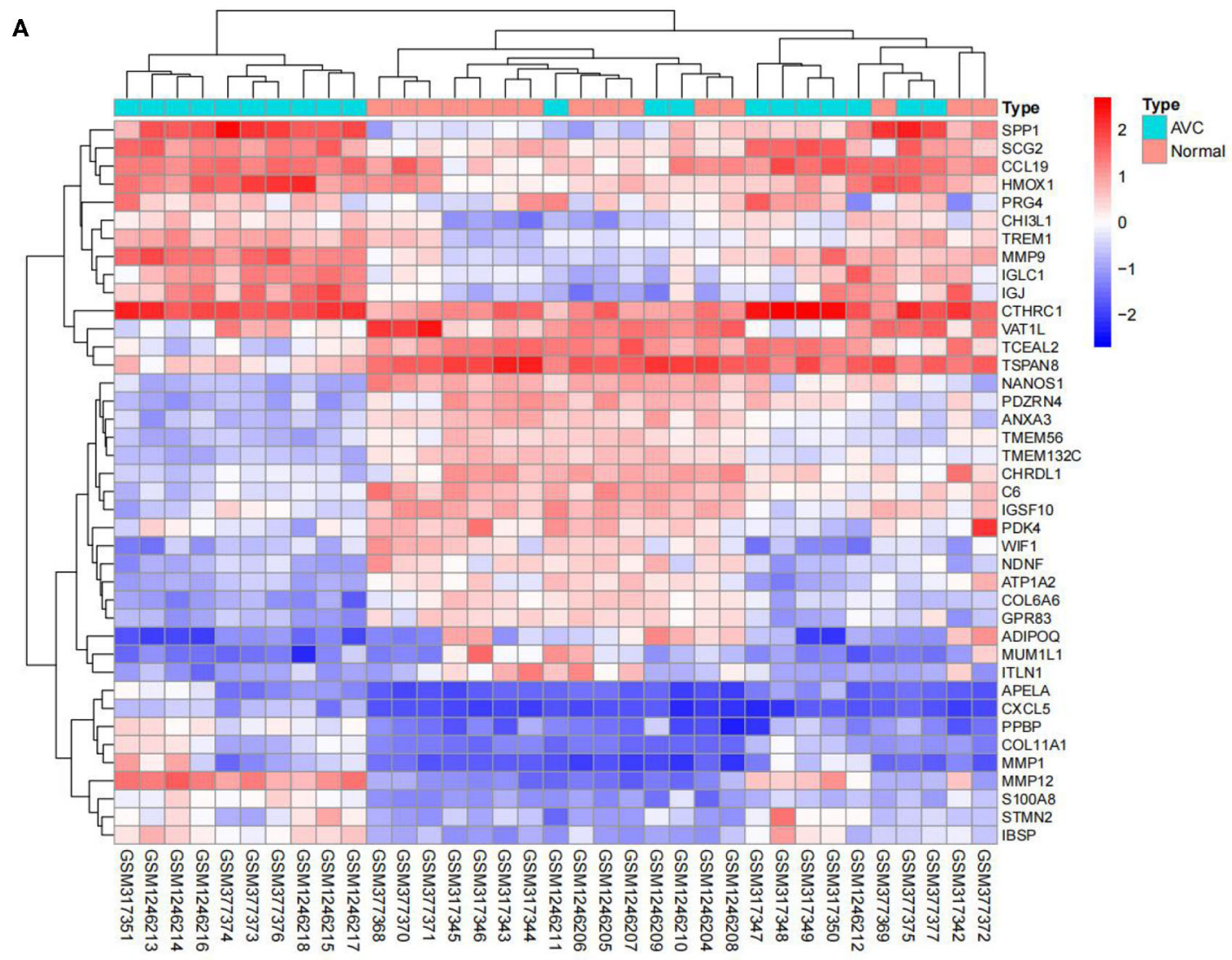

B

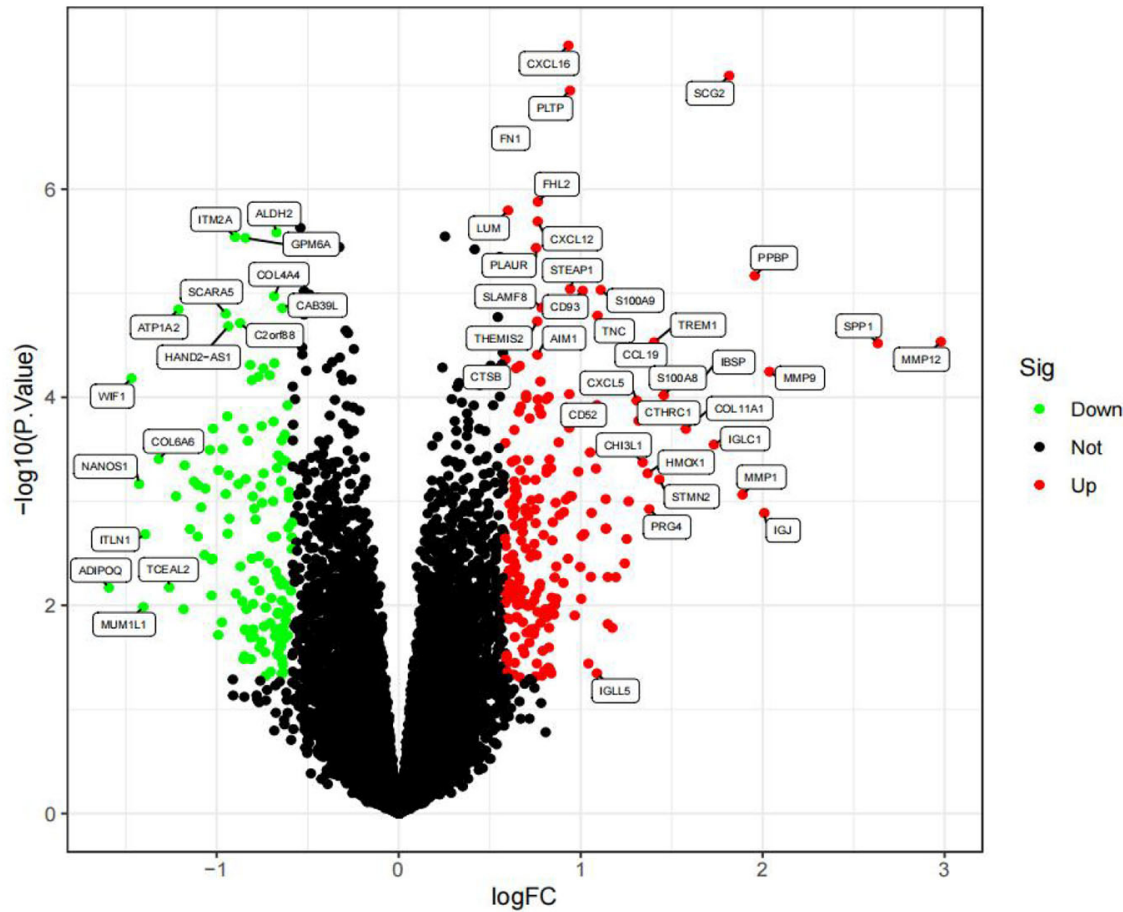

FIGURE 2 | Visualizing the results of differential genes. (A) Clustering heatmap of the genes exhibiting significantly differential expression AVC vs. normal group. Statistically significant DEGs were defined as $\| \log 2 F o l d c h a n g e \mid>0.585$ and $P$-value $<0.05$. DEG, differentially expressed gene; AVC, Aortic valve calcification group. Cyan represents AVC groups; red-orange represents normal groups. (B) Volcano map of DEGs; red represents up-regulated differential genes, black represents no significant difference genes, and green represents down-regulated differential genes. 
TABLE 1 | The top 10 up- and down-regulated DEGs in AVC and normal sample.

\begin{tabular}{lcc}
\hline Gene symbol & Fold-change & $\boldsymbol{P}$-value \\
\hline Top 10 up-regulated DEGs & & \\
MMP12 & 2.979149817 & $2.92 \mathrm{E}-05$ \\
SPP1 & 2.632830567 & $3.03 \mathrm{E}-05$ \\
MMP9 & 2.037460333 & $5.66 \mathrm{E}-05$ \\
IGJ & 2.008886783 & 0.001292002 \\
PPBP & 1.956678033 & $6.77 \mathrm{E}-06$ \\
MMP1 & 1.889498133 & 0.000862519 \\
SCG2 & 1.815960233 & $8.12 \mathrm{E}-08$ \\
IGLC1 & 1.732297767 & 0.000284768 \\
IBSP & 1.645398033 & $7.50 \mathrm{E}-05$ \\
COL11A1 & 1.577731517 & 0.000201496 \\
Top 10 down-regulated DEGs & & \\
ADIPOQ & -1.5926205 & 0.006759383 \\
WIF1 & -1.46850865 & $6.57 \mathrm{E}-05$ \\
NANOS1 & -1.426355333 & 0.000681789 \\
MUM1L1 & -1.403391017 & 0.010340331 \\
ITLN1 & -1.393083817 & 0.002074118 \\
COL6A6 & -1.318182983 & 0.000392129 \\
TCEAL2 & -1.260862617 & 0.006721387 \\
TSPAN8 & -1.223407683 & 0.000891201 \\
ATP1A2 & -1.209701467 & $1.43 \mathrm{E}-05$ \\
PDZRN4 & -1.1820793 & 0.010880592 \\
\hline & &
\end{tabular}

of the findings of the PCA cluster analysis, it was discovered that there was a statistically significant difference in immune cell infiltration (Figure 9A). We compared the composition of immune cell infiltration in AVC and normal samples using the data matrix of the combined datasets of GSE12644 and GSE51472 (Figure 9C). The study's findings indicated that the fraction of naïve B cells $(P<0.05)$, activated natural killer cells $(P<0.05)$, and monocytes $(P<0.05)$ was significantly higher in normal tissues than in AVC tissues. However, in normal tissues, the ratio of memory B cells $(P<0.05)$, plasma cells $(P<0.05)$, and macrophages M0 $(P<0.05)$ was much lower than in AVC tissues (Figure 9D). Additionally, the relationship between 22 immune cells was analyzed (Figure 9B). Naïve B cells strongly positively connected with activated natural killer cells $(r=0.32)$, but substantially negatively associated with macrophages $\mathrm{M} 0(\mathrm{r}$ $=-0.31)$, plasma cells $(r=-0.36)$, and monocytes $(r=-0.18)$. Memory B cells were strongly positively connected with with plasma cells $(r=0.06)$ and macrophages M0 $(r=0.29)$, but substantially negatively associated with naïve $B$ cells $(r=-0.25)$, monocytes $(r=-0.25)$, and activated natural killer cells $(r=$ -0.27 ). Plasma cells were strongly positively connected with memory B cells $(r=0.06)$ and macrophages M0 $(r=0.05)$, but substantially negatively associated with naïve $B$ cells $(r=-0.36)$, monocytes $(\mathrm{r}=-0.01)$, and activated natural killer cells $(\mathrm{r}=$ -0.11 ). Activated natural killer cells were strongly positively connected with naïve B cells $(r=0.32)$ and monocytes $(r=$ 0.38 ), but substantially negatively associated with memory $\mathrm{B}$ cells $(r=-0.27)$, macrophages M0 $(r=-0.34)$, and plasma cells $(\mathrm{r}=-0.11)$. Monocytes were strongly positively connected with activated natural killer cells $(\mathrm{r}=0.38)$, but substantially negatively associated with naïve $B$ cells $(r=-0.18)$, memory $B$ cells $(r=-0.25)$, plasma cells $(r=-0.01)$, and macrophages M0 $(r=-0.35)$. Macrophages M0 were strongly positively connected with memory B cells $(r=0.29)$ and plasma cells $(r$ $=0.05$ ), but substantially negatively associated with naïve $\mathrm{B}$ cells $(r=-0.31)$, activated natural killer cells $(r=-0.34)$, and monocytes $(\mathrm{r}=-0.35)$.

\section{FN1 and Infiltrating Immune Cells}

We analyzed the correlation of the above results of immune infiltration with FN1. As shown in Figure 10A, FN1 was positively correlated with memory B cells $(\mathrm{r}=0.40, P=0.0018$; Figure 10B), macrophages $\mathrm{M} 0(\mathrm{r}=0.44, P=0.008$; Figure 10C) and activated mast cells $(\mathrm{r}=0.45, P=0.007$; Figure 10D), but significantly negatively correlated with resting mast cells $(\mathrm{r}=$ $0.39, P=0.021$; Figure 10E), monocytes $(\mathrm{r}=-0.35, P=0.040$; Figure 10F) and activated natural killer cells $(\mathrm{r}=-0.58, P=$ 0.0003; Figure 10G). The correlation between FN1 and immune cells is presented in Supplementary File 7.

\section{Identification of Small Molecule Drugs}

DEGs were first separated into up-regulated and down-regulated categories, and then enriched with substantially altered genes acquired from treatment with small compounds from the CMap database. As part of the AVC tissue study, yohimbic acid was identified as one of the expected small compounds that might block the expression of genes related with AVC (Table 2).

\section{DISCUSSION}

AVC is a term that refers to a collection of aortic valve diseases ranging from calcification to hardness to stenosis (31). Its occurrence rises as a person ages, affecting $\sim 9 \%$ of patients over the age of 80 years. Aortic sclerosis is more prevalent, affecting $25-30 \%$ of people over the 65 years old and progressing at a rate of around $2 \%$ every year to aortic valve stenosis (32). AVC is a complex and dynamic process that is regulated by a variety of physiological and pathological factors such as lipoprotein deposition, inflammatory response, activation of renin-angiotensin-aldosterone, extracellular matrix transformation, and cellular ossification $(33,34)$, thereby destroying valve-specific cells including endothelial cells as well as interstitial cells, aggravating valvular calcification, causing valve orifice stenosis, and obstructing the left ventricular outflow path (35). For advanced AVC or AVC accompanied by significant clinical symptoms, surgery and transcatheter aortic valve replacement are presently the most effective therapies. However, these procedures are associated with increased costs, risk of mortality, and perioperative and postoperative adverse events including anticoagulation-related bleeding or thrombotic events, and reoperation owing to artificial valve insufficiency (4). Thus, the importance of expanding our understanding of the molecular mechanism of degenerative cardiac valve diseases is emphasized $(36,37)$. Gene sequencing and bioinformatics technology have advanced substantially in recent decades, making it feasible to 
A

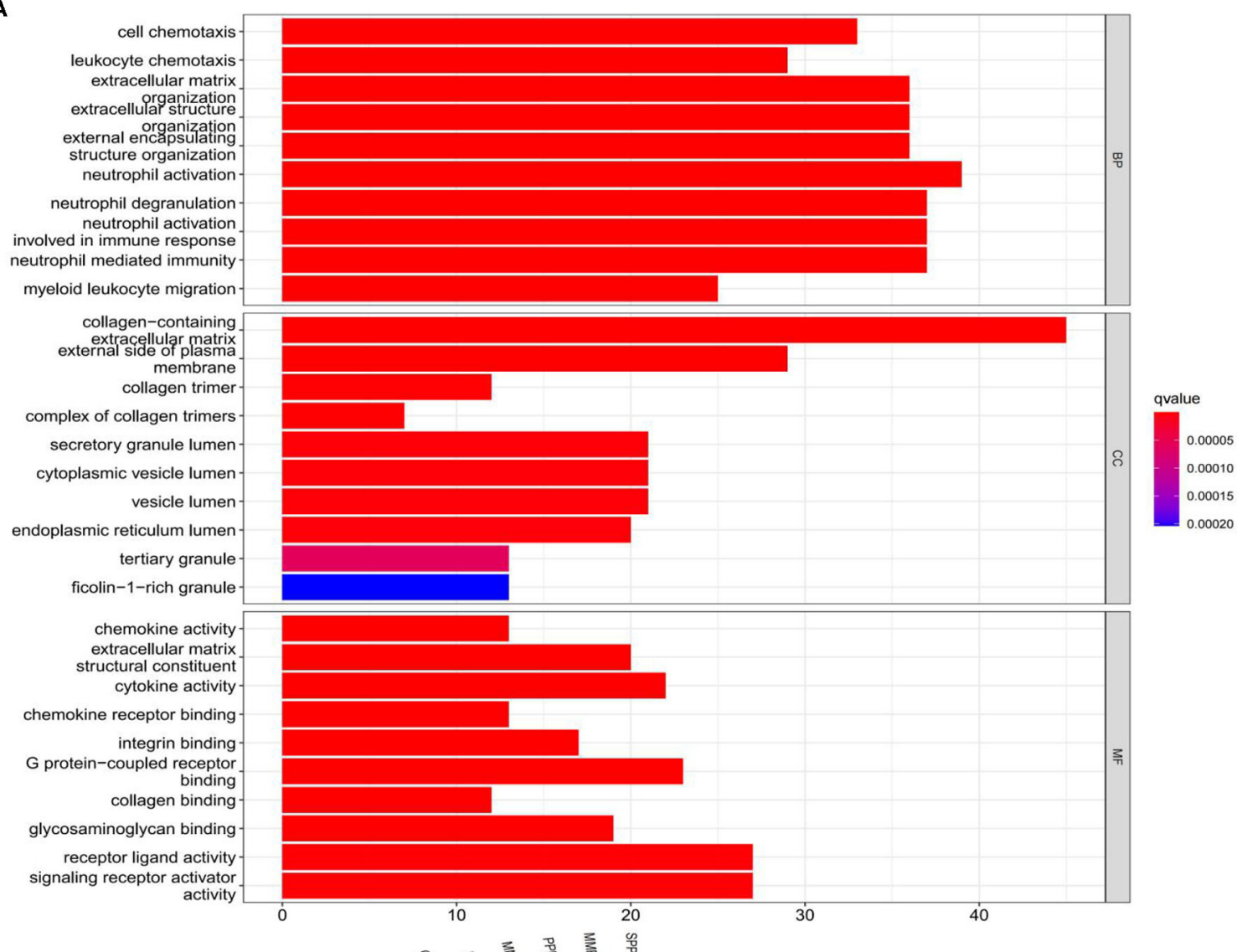

B

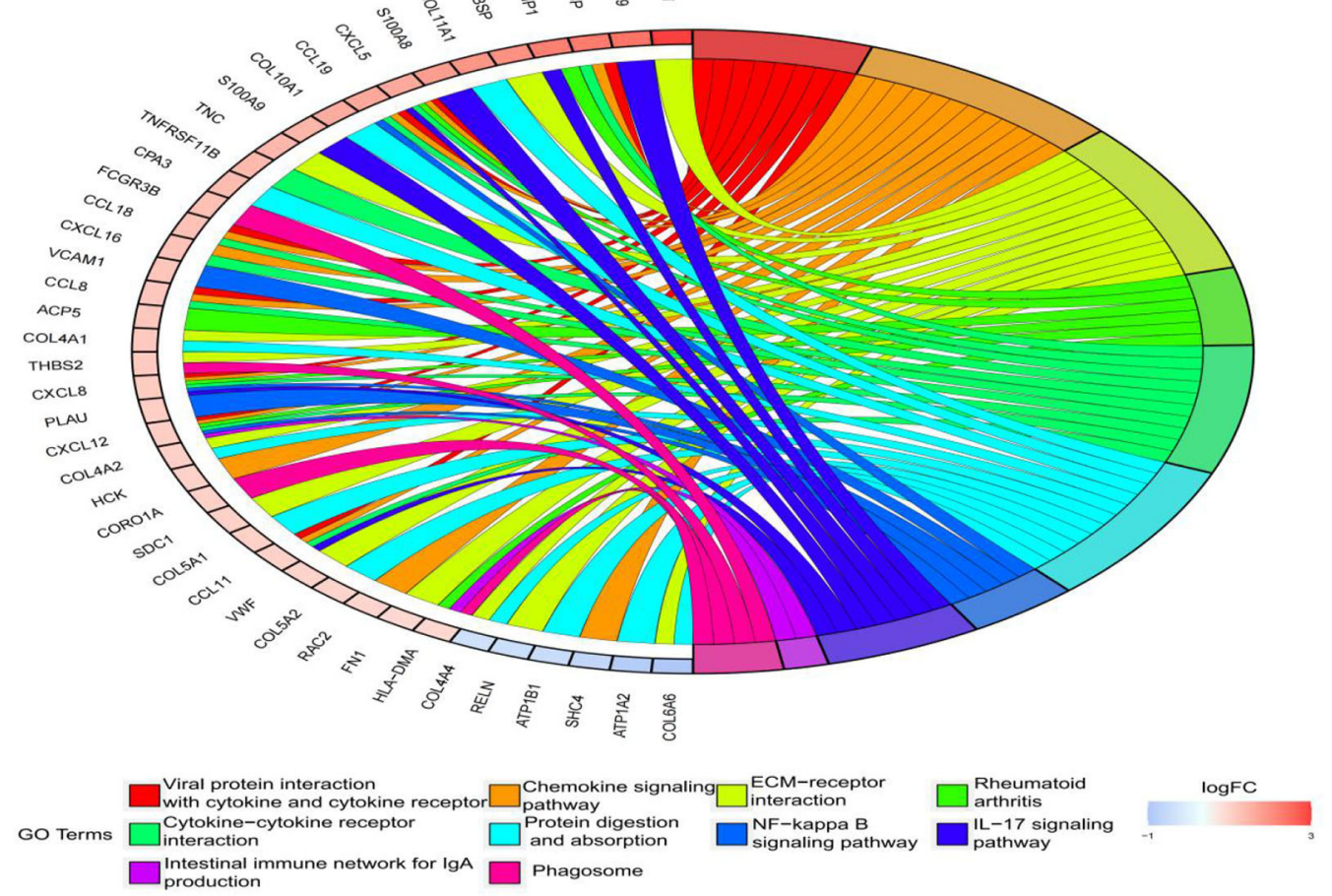

FIGURE 3 | Functional enrichment analyses of DEGs. (A) Gene Ontology (GO) enrichment analyses of DEGs. The x-axis shows the number of genes enriched on the terms, and the $y$-axis shows the pathway terms. The q-value of each term is colored according to the legend. BP, biological process; CC, cellular component; MF, molecular function; (B) Kyoto Encyclopedia of Genes and Genomes (KEGG) enrichment analyses of DEGs. Select the first 30 DEGs to connect to the enriched pathway terms. The q-value of each term is colored according to the legend. The different colors represent different pathway terms. 


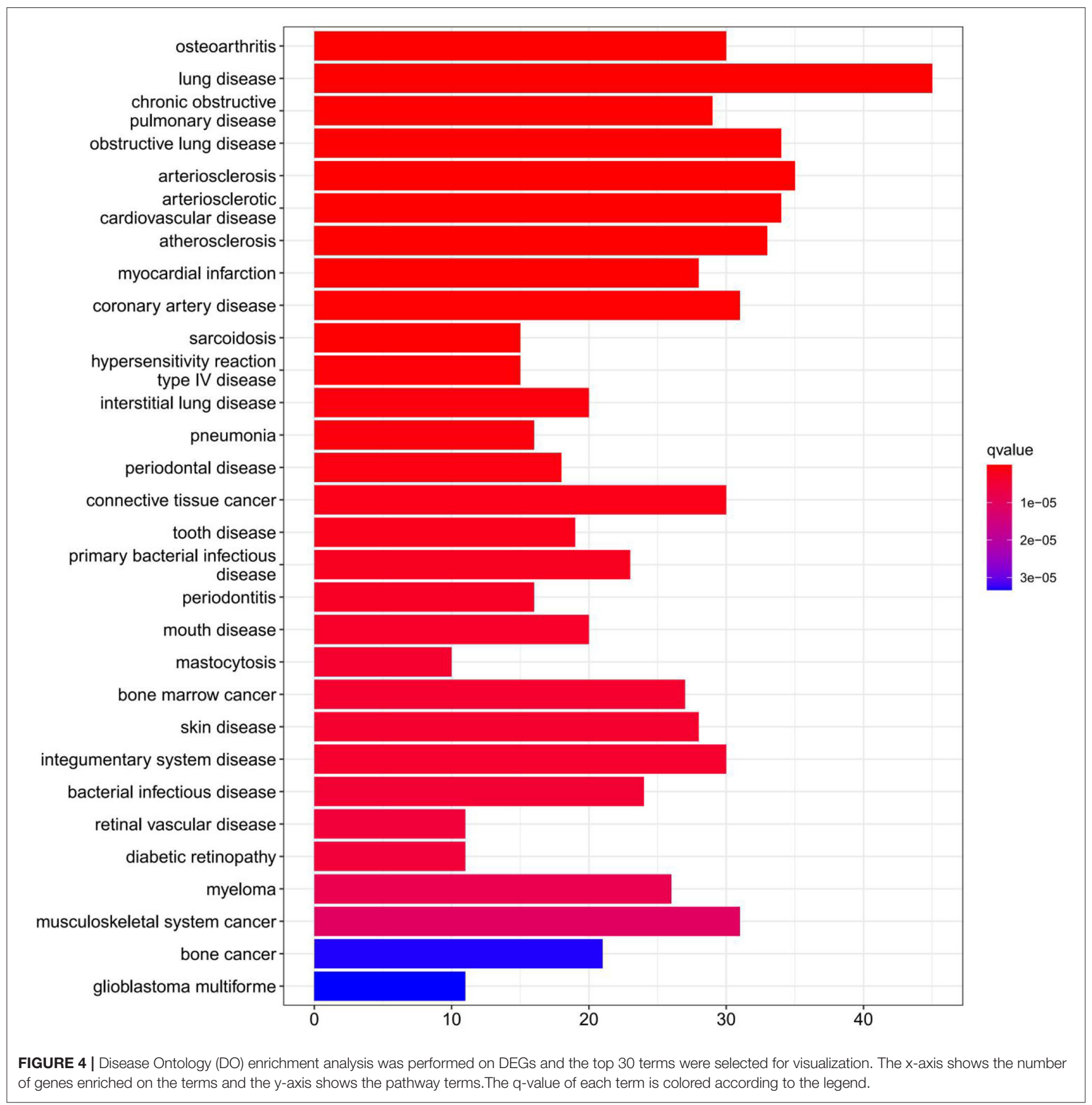

evaluate and utilize massive amounts of sequencing data (38). Nevertheless, few studies have examined the association between abnormally expressed genetic markers and immunological invasion in AVC vs. normal tissues. Therefore, through the analysis of AVC and normal tissues, we try to identify potential diagnostic biomarkers and further observe the role of immune cell infiltration in the disease.

To our information, compared to previous studies (39, 40), the analytical approach to filtering the biomarkers of the AVC was first investigated using the lasso and SVM algorithms. Lasso logistic regression, a machine learning method, identifies variables by selecting those with the lowest probability of classification error (41). SVM-RFE is a machine learning technique that has been used in ranking features and selecting the most important features for classification in multiple applications (42). Recently, Akter et al. (43) found that combining multiple machine learning methods could improve predictive performance and thus provide a highly accurate predictive diagnostic model. Thus, taking advantage of the two machine learning methods is beneficial for us to discover 


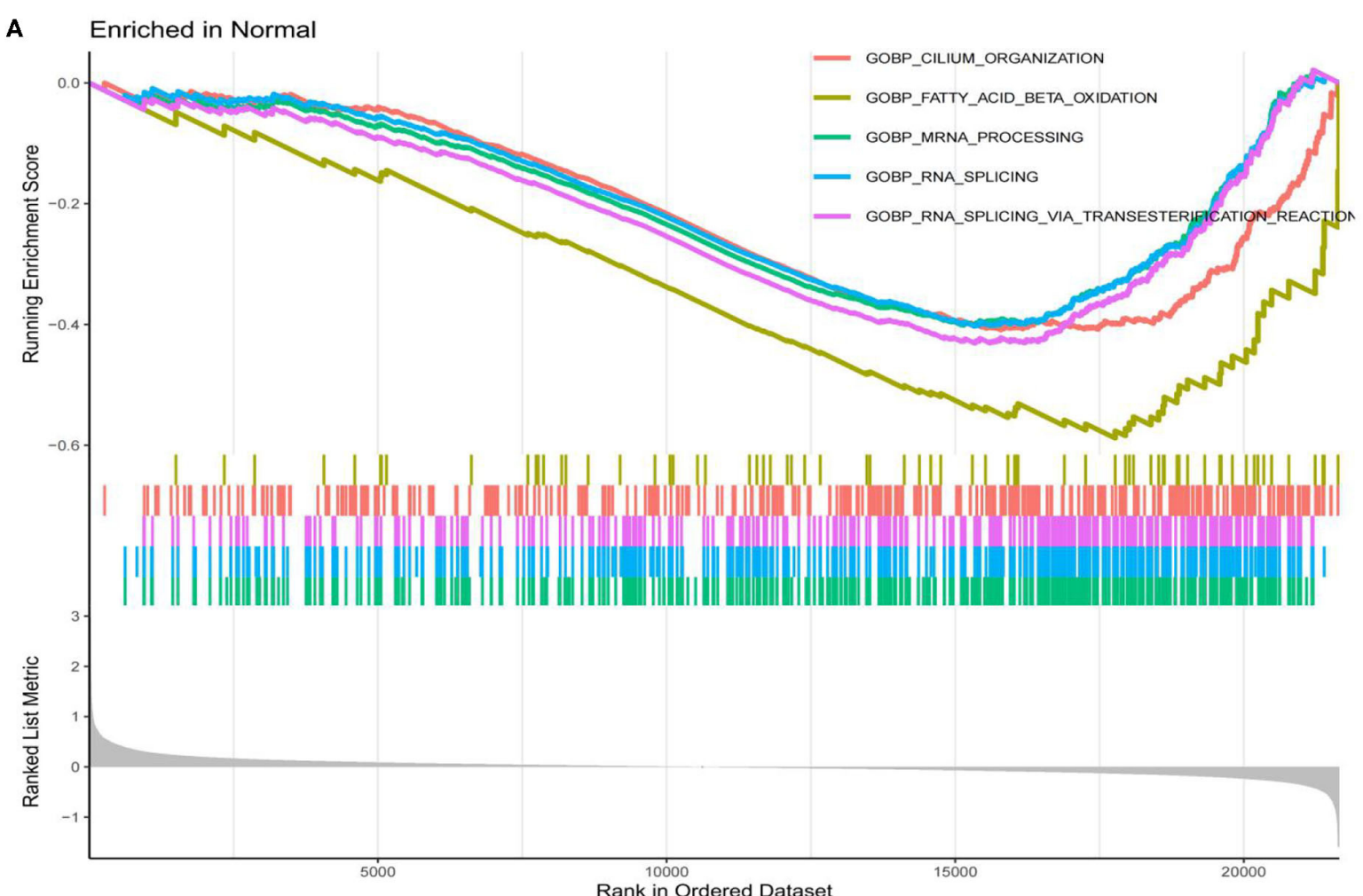

B

Rank in Ordered Dataset

B Enriched in AVC

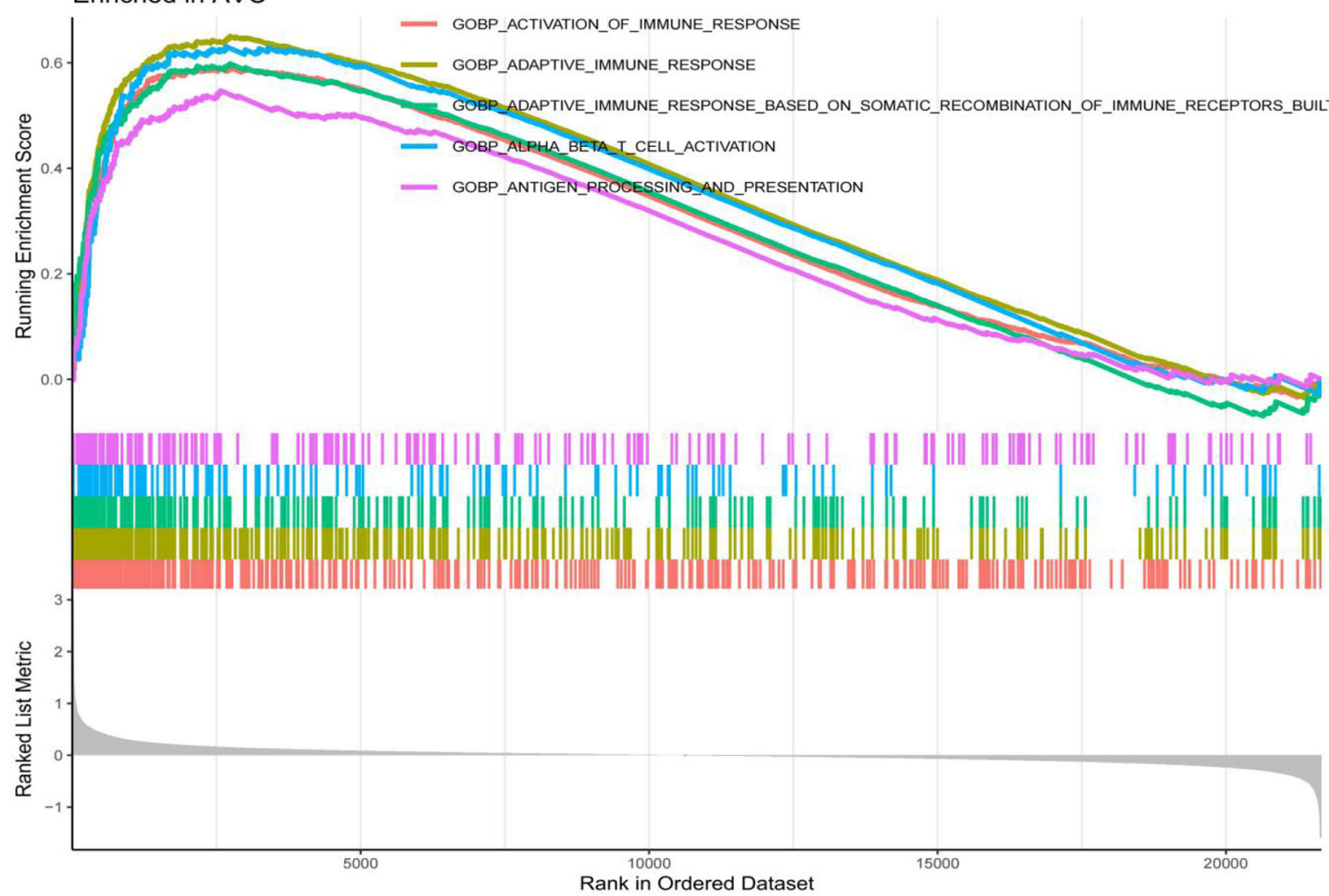

FIGURE 5 | LogFC values were calculated for all genes, and Gene set enrichment analysis (GSEA) analysis was performed based on logFC using c2.cp.kegg.v7.4.symbols.gmt and c5.go.v7.4.symbols.gmt in the normal and AVC groups using. (A) Analysis of the GO pathway terms for all genes enriched in the normal group using GSEA. (B) Analysis of the GO pathway terms for all genes enriched in the AVC group using GSEA. 
A
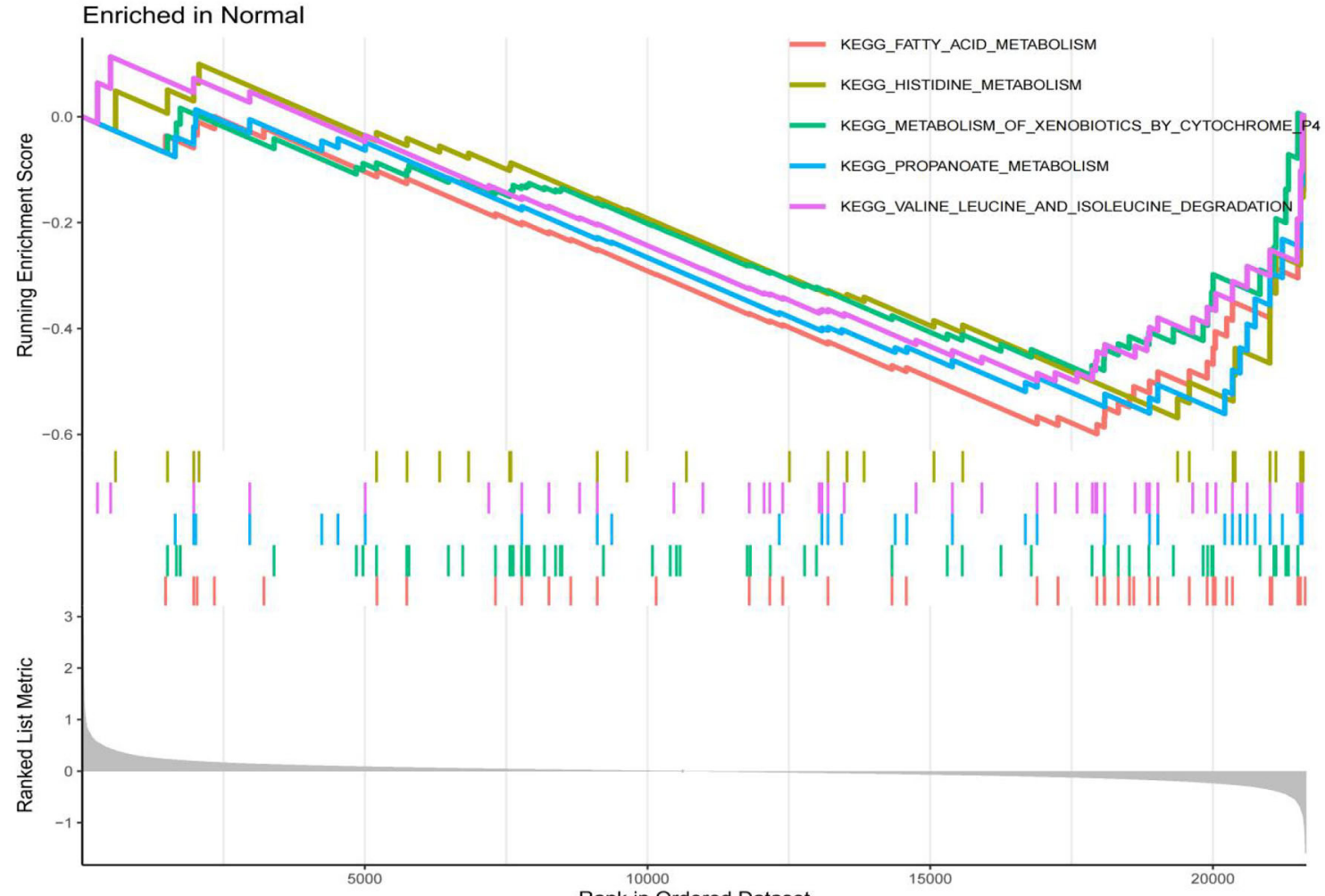

B

Rank in Ordered Dataset

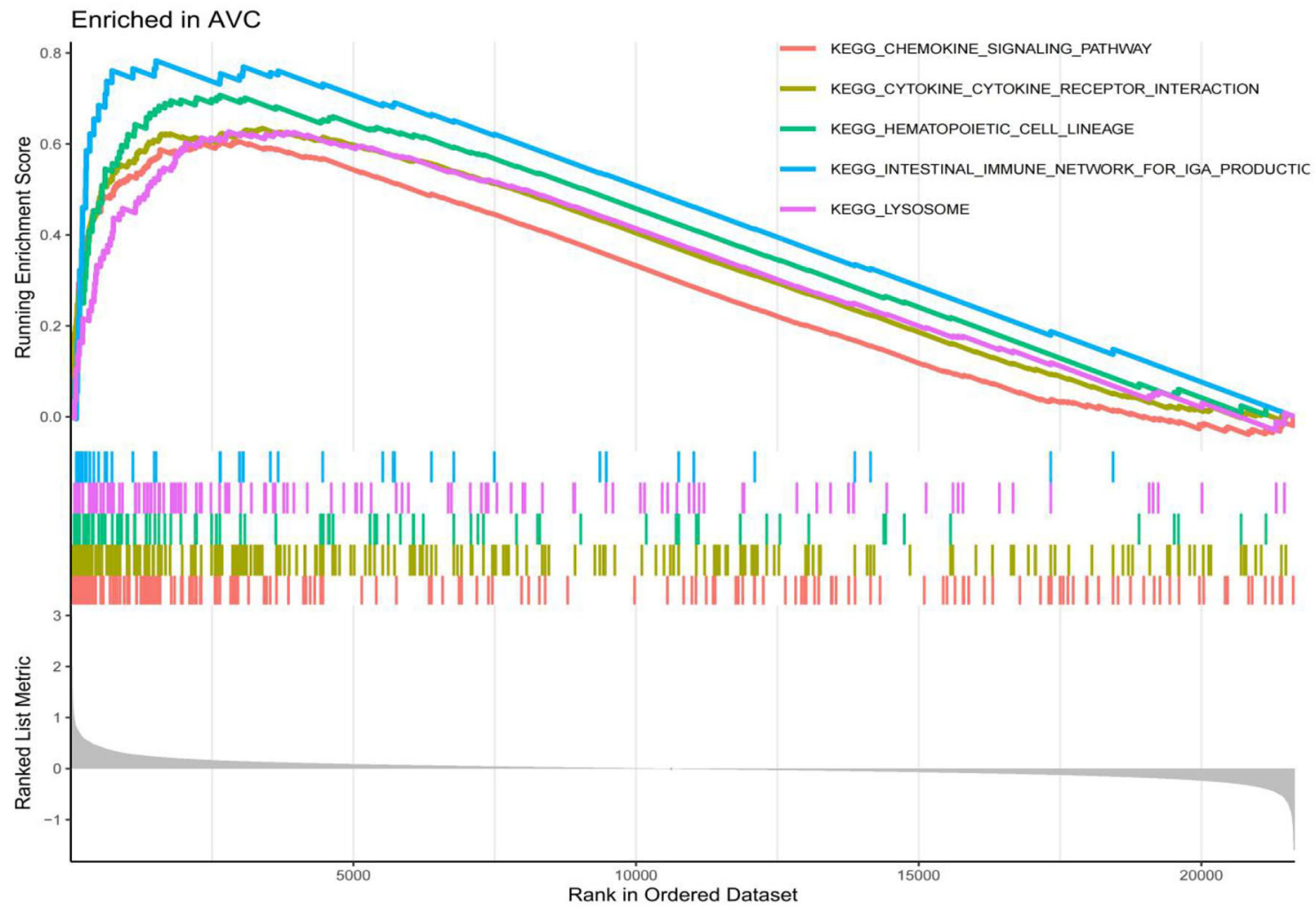

FIGURE 6 | Analysis of the top 4 Kyoto Encyclopedia of Genes and Genomes (KEGG) pathway terms for all genes enriched in the normal and AVC groups using Gene set enrichment analysis (GSEA). (A) Analysis of the KEGG pathway terms for all genes enriched in the normal group using GSEA. (B) Analysis of the KEGG pathway terms for all genes enriched in the AVC group using GSEA. 


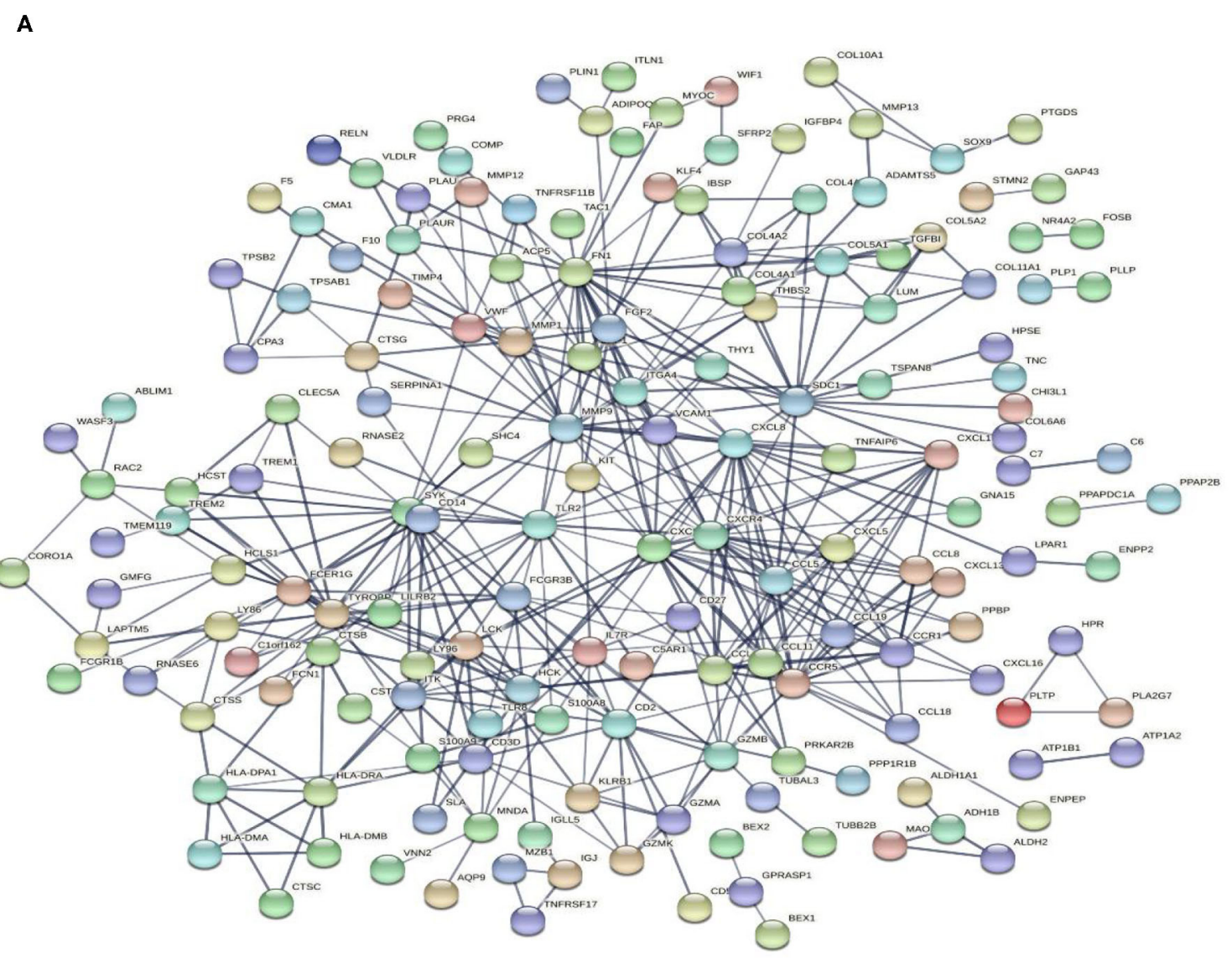

B

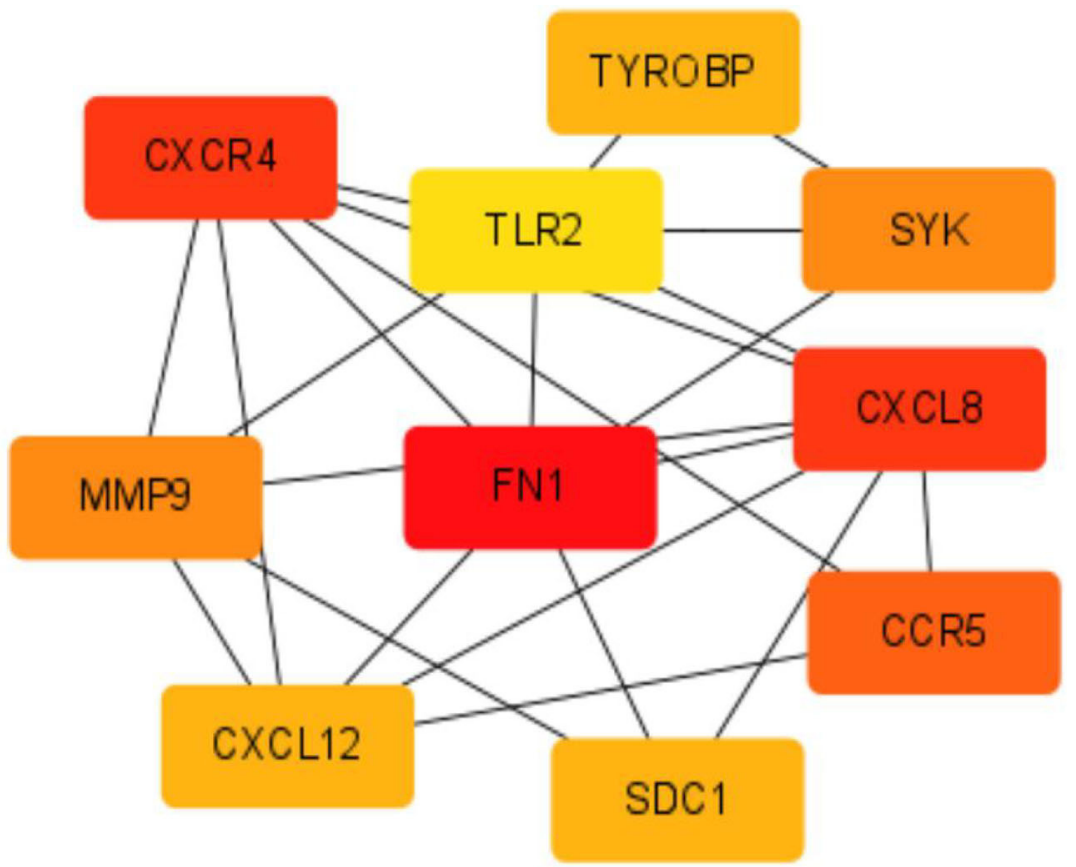

FIGURE 7 | PPIN and hub gene identification. (A) PPIN was constructed by all the 337 DEGs using STRING database. The protein-protein interactions are represented by linkages. The number of nodes: 320; the number of edges: 420; the average node degree: 2.62; the PPI enrichment $p$-value $<1.0$ e-16. (B) The top 10 hub genes in the PPIN were screened by Cytoscape (v3.6.1) plugin cytoHubba based on their connectivity degree. The 10 identified hub genes such as FN1, CXCL8, CXCR4, CCR5, MMP9, SYK, TYROBP, CXCL12, SDC1, and TLR2 are displayed from red (high degree value) to yellow (low degree value). PPIN, protein-protein interaction network; DEG, differentially expressed gene; STRING, search tool for the retrieval of interacting genes. 
A

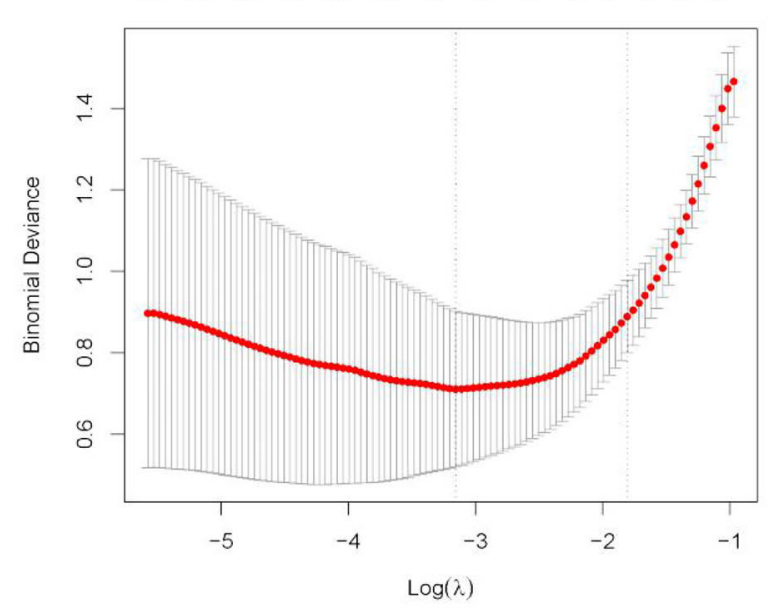

c

SVM-RFE

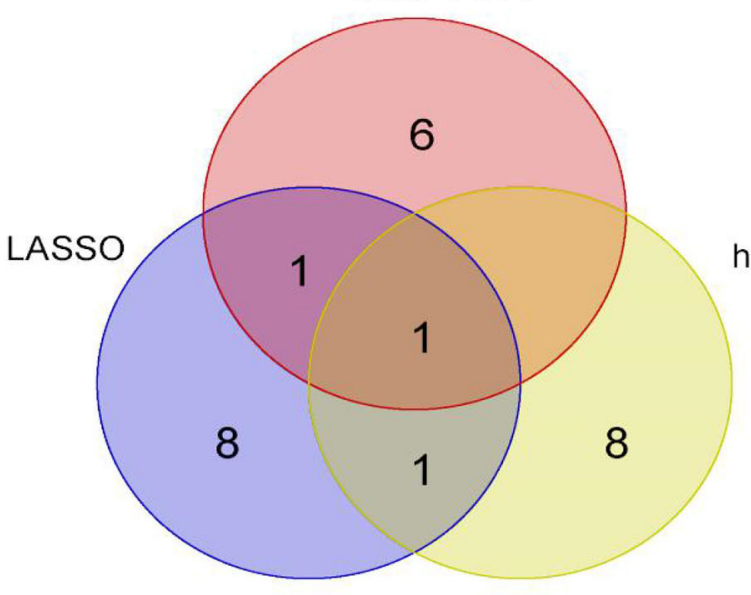

E

审Normal

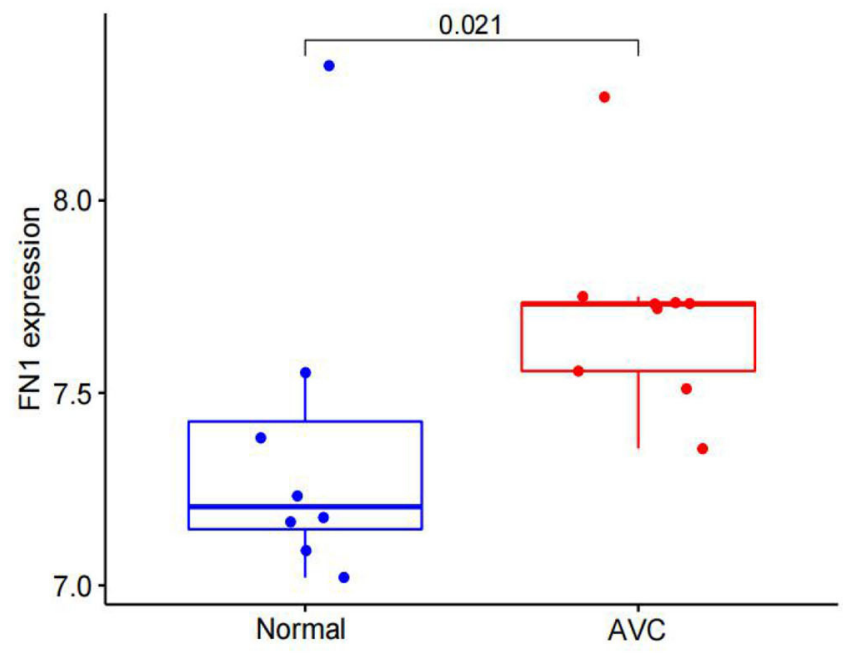

B

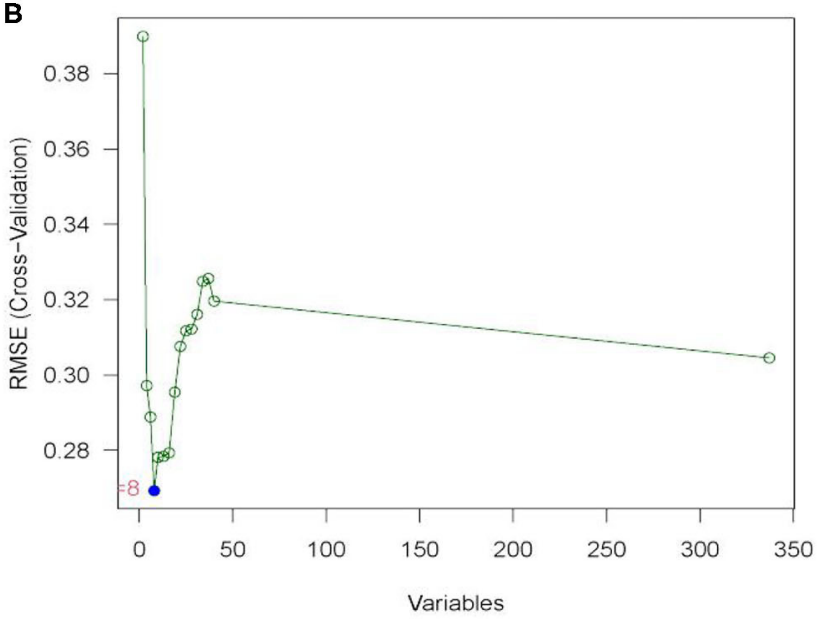

D

FN1

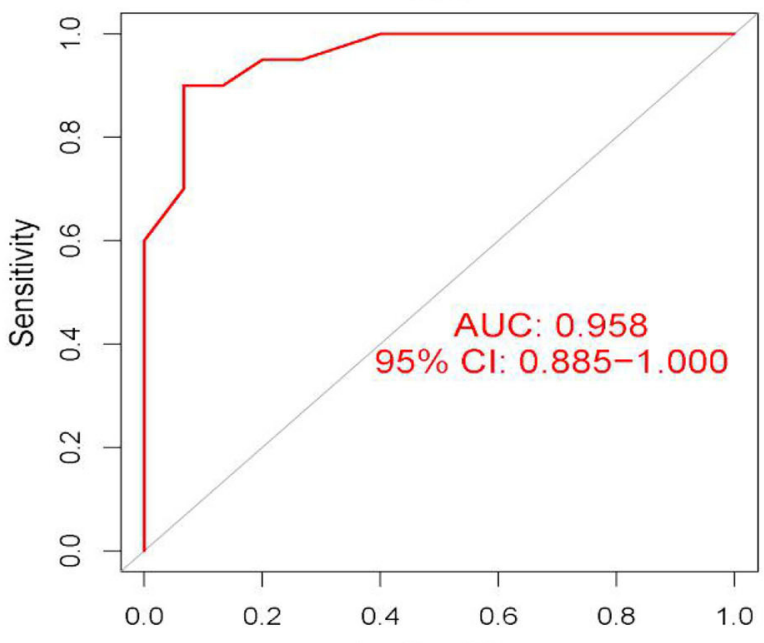

FN1

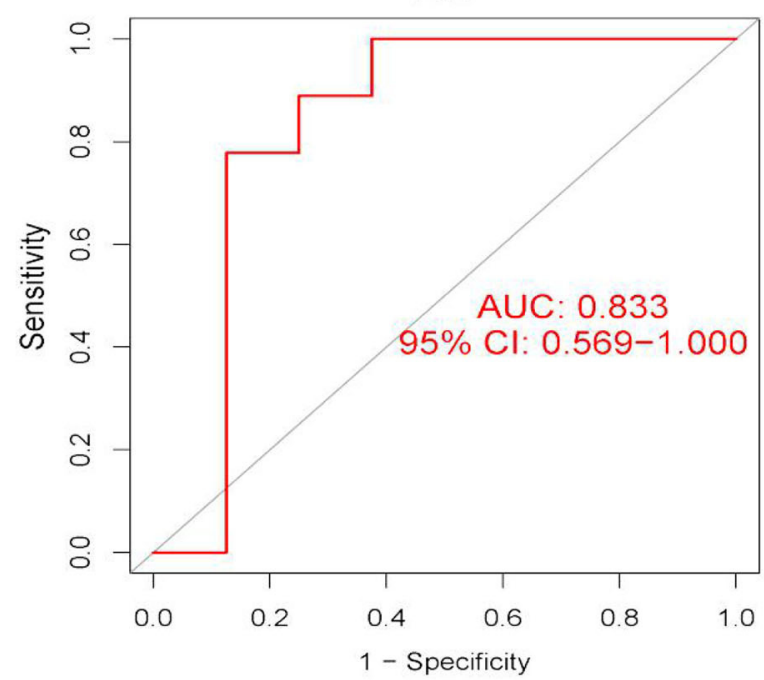

FIGURE 8 | Screening and verification of diagnostic markers. (A) Tuning feature selection in the least absolute shrinkage and selection operator (LASSO) model. The DEGs were narrowed down using the LASSO regression algorithm, resulting in the identification of 11 variables as diagnostic biomarkers for AVC. The ordinate is the value of the coefficient, the lower abscissa is $\log (\lambda)$, and the upper abscissa is the number of non-zero coefficients in the model at this time. (B) A plot of biomarkers (Continued) 
FIGURE 8 | selection via support vector machine-recursive feature elimination (SVM-RFE) algorithm. A subset of five features among the DEGs was determined using the SVM-RFE algorithm. (C) Venn diagram demonstrating one diagnostic marker shared by the least absolute shrinkage and selection operator and SVM-RFE algorithms and hub genes. (D) The diagnostic performance of the calculated based on the FN1 expression in AVC diagnosis in training data set. (E) FN1 mRNA expression in AVC compared to normal groups in the validation set. (F) The diagnostic performance of the calculated based on the FN1 expression in AVC diagnosis in test data set. The distinction was considered good when the AUC value was between 0.8 and 0.9 , and exceptional when the AUC value was $>0.9$. ROC, receiver operating characteristic; AUC, area under the ROC curve.

potentially important biomarkers, which is very meaningful for our research on AVC. We collected two cohorts from the GEO datasets and analyzed the data in an integrated manner. A total of 337 DEGs were discovered, comprising 203 upregulated and 134 down-regulated genes. Diseases enriched by DEGs, according to the results of enrichment analyses, were mostly associated with arteriosclerosis and arteriosclerotic cardiovascular disease. Following analysis, it was shown that the enriched pathways were mostly associated with the inflammation and immunological response, such as cytokine-cytokine receptor interactions and chemokine signaling. These results corroborate previous findings that inflammation plays a role in the development and progression of $\operatorname{AVC}(44,45)$ and that AVC is an inflammation-dependent process (46). Initially thought to be a "degenerative" procedure, AVC is now identified as an active condition in which interplay between valvular interstitial cells, the major biological constituent of the aortic valve, and circulatory inflammatory cells and endothelial progenitor cells drive tissue restructuring (47). Numerous findings revealed the presence of many pro-inflammatory cytokines, including tumor necrosis factor, oxidized low-density lipoprotein cholesterol, and interleukin (IL)-1/2/6, in calcified valves $(48,49)$.

Inflammation is speculated to play a role in the etiology of AVC, with T lymphocytes (50) and macrophages (10) being found in early aortic valve lesions. IL-6 has been reported to perform as a proinflammatory factor, activating biomineralization and osteogenic signaling mechanisms in the cardiovascular system $(11,44,51,52)$. This result is congruent with our findings, validating the credibility of the current study's findings and indicating the critical role of the immune response in AVC. The immunological response is perhaps the most varied and complicated reaction that occurs after the activation of inflammatory processes within the aortic valve, and it has been shown to impact various calcification processes (53). Furthermore, to provide a safe and effective therapy, it is also vital to precisely regulate development of various immune cell types in aortic valve. Thus, novel biomarkers of disorders associated to immune cell infiltration may be discovered using bioinformatics analysis to block related pathways to improve AVC therapy.

Based on two machine-learning algorithms and hub gene identification, one diagnostic marker was identified. Fibronectin is a glycoprotein ligand that is extensively expressed in a variety of cell types that bind cell surfaces and compounds including collagen, fibrin, heparin, DNA, and actin (54). FN1 belongs to this family and plays a role in a variety of biological activities, including cell migration, adhesion, and cytoskeleton structure (55). Aberrant FN1 expression is also associated with a multitude of diseases, including cancer, atherosclerosis, and arthritis (56-58). A previous study showed that osteoblasts are accompanied by type I collagen production during proliferation and differentiation, and found high expression of FN1, implying that osteoblasts are accompanied by upregulation of FN1 during active bone formation (58). Moreover, FN1 is engaged in osteoblast compaction through matrix assembly processes mediated by FN fibrillogenesis cells, which are essential for osteoblast mineralization (59). Yang et al. (60) have recently discovered $\mathrm{FN} 1$ as a molecular regulator in promoting osteogenic differentiation in vitro. In vitro, $\mathrm{FN}-$ 1 knockdown decreases $\beta$-catenin expression and inhibits $\mathrm{WNT}$ /-catenin signaling, hindering osteoblast differentiation and mineralization., while overexpression of FN1 stimulates $\mathrm{WNT} / \beta$ catenin signaling and promotes pre-osteoblast differentiation and mineralization (60).As AVC advances, inflammatory cells inside the subendothelium and fibrosa cause oxidative stress and generate a variety of growth factors and cytokines (11), including transforming growth factors, tumor necrosis factors, interleukin-1b (IL-1b), and receptor activator of nuclear factor$\kappa \mathrm{B}$ ligand. These growth factors and cytokines are major inducers of valve osteoblast development by activating multiple signaling pathways (including Notch, bone morphogenetic proteins and $\mathrm{WNT} / \beta$-catenin) and establishing an environment conducive to osteogenesis (61). In this study, we believe that by stimulating the $\mathrm{WNT} / \beta$-catenin signaling pathway, FN1 may play a critical role in the development of osteogenesis in calcified aortic valve disease. In addition, Jun et al. (62) revealed that the expression and release of Fn had a substantial impact on the effects of ATP and caspase-1 generated by inflammasome activators. Moreover, As an endogenous danger signal, it boosts the inflammatory process by stimulating caspase1 and resulting in the death of inflammatory cells. The high concentration of FN-fibrin complex in plasma can promote the release of inflammatory factors from tissues to the circulation during the progression of a variety of diseases, including thrombosis, plaque formation, the development and progression of atherosclerosis, and aging arteries Wall lesions, which further aggravates the inflammatory response and promotes disease progression (63). FN-fibrin complexes have been associated with numerous diseases, including diabetes mellitus (64), rheumatoid arthritis (65), osteoarthritis (66), chronic obstructive pulmonary disease (67), and coronary artery disease (68). Thus, our findings suggest that the FN1 may be critical in the evolution of the inflammatory process in calcified aortic valve disease.

By using CIBERSORT to analyze the immune cell types between AVC and normal samples, it is found that the basic biological processes related to AVC are closely related to a variety of immune cells. The analysis results found that memory B cells and macrophages M0 are significantly expressed in AVC 


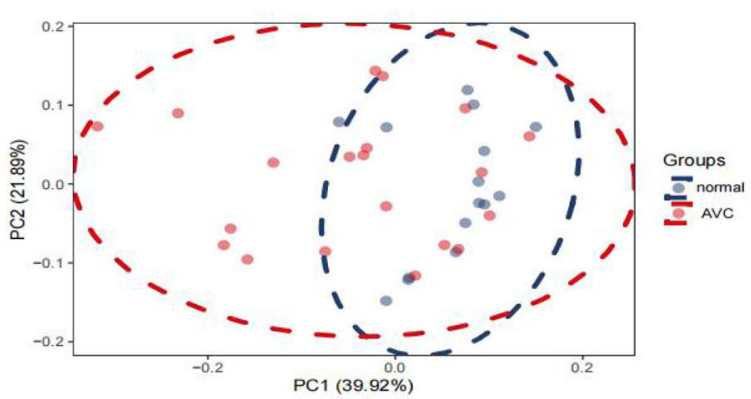

C

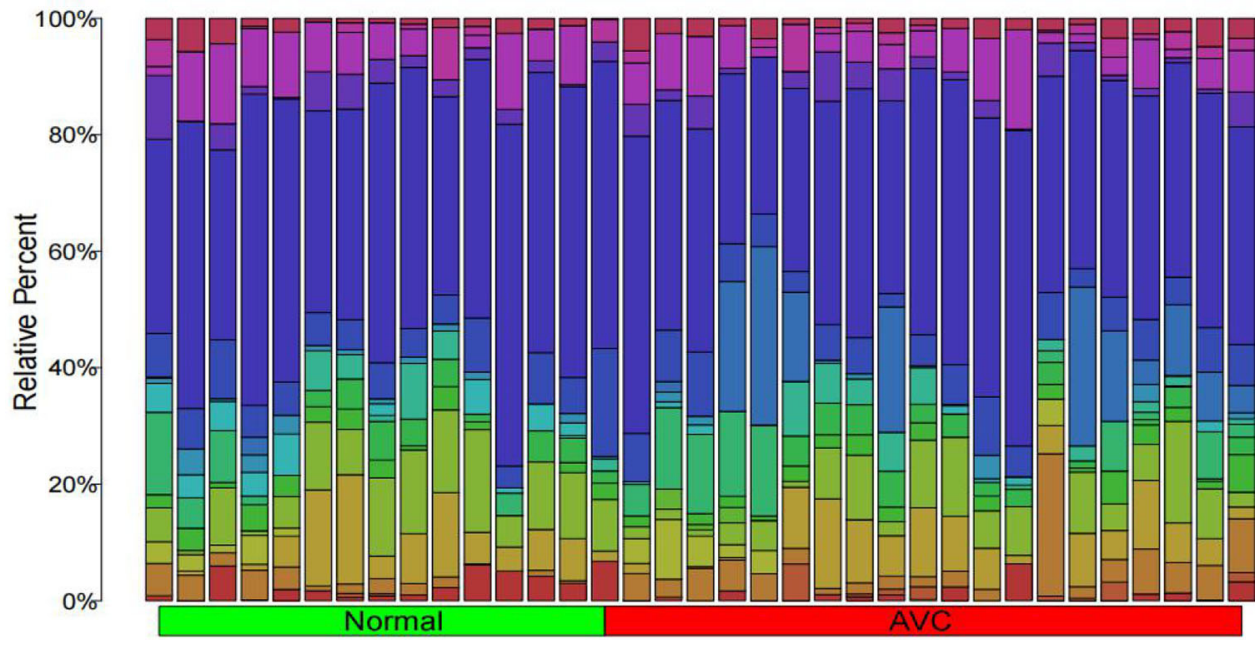

B
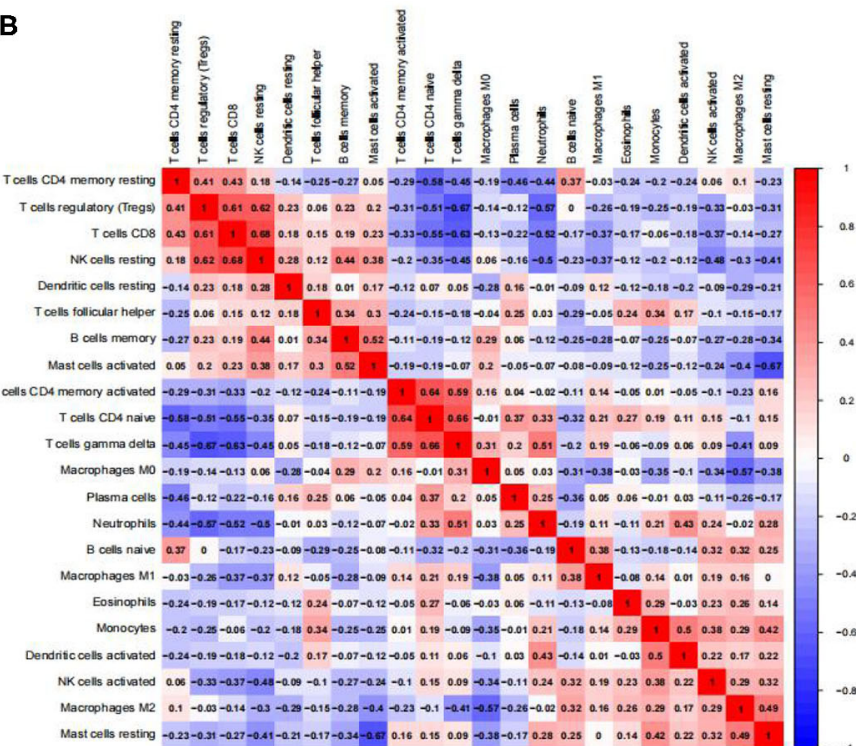

D

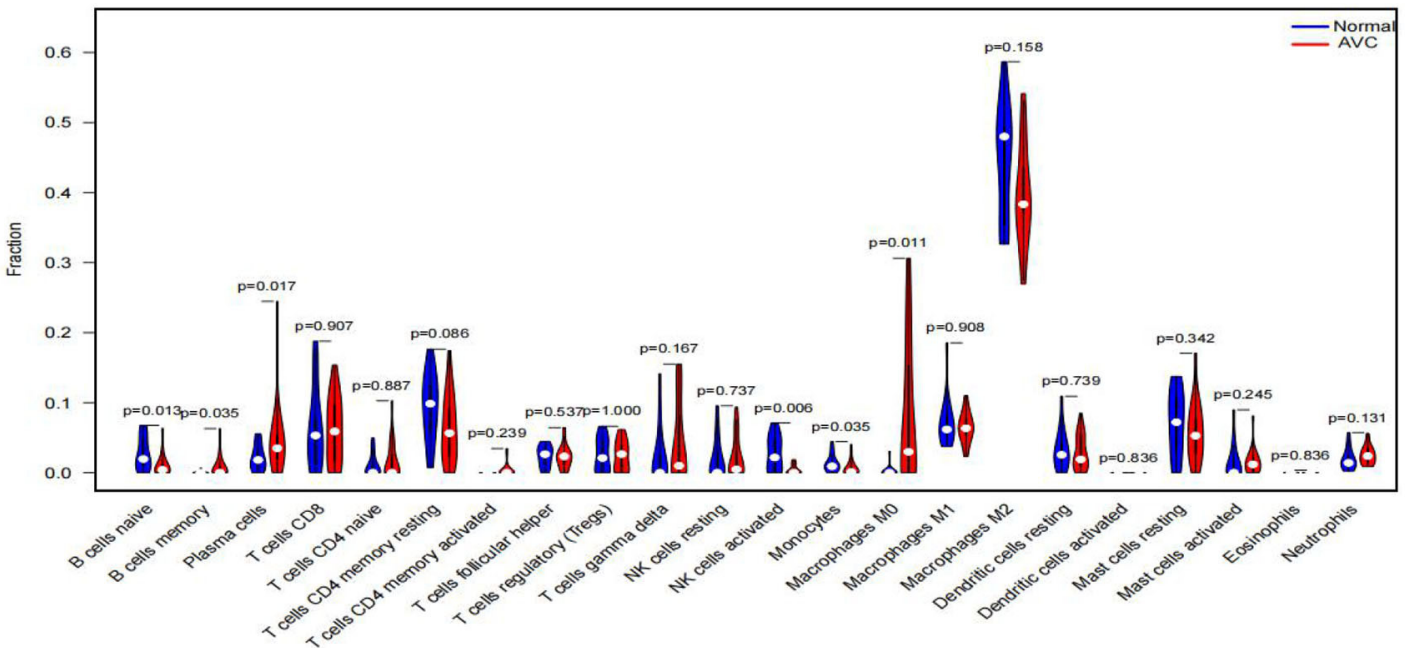

FIGURE 9 | Comparing the composition of immune cell infiltration in the normal and AVC samples by using the combined data matrix of GSE12644 and GSE51472 and visualized the results. (A) PCA cluster plot of immune cell infiltration between normal and AVC groups. (B) Correlation heat map of 22 types of immune cells. The size of the colored squares represents the strength of the correlation; red represents a positive correlation, blue represents a negative correlation. The redder the color, the stronger the correlation. (C) The heat map of the 22 subpopulations of immune cells. (D) Violin diagram of the proportion of 22 types of immune cells. (The normal controls group was marked as blue color and AVC group was marked as red color. $P$-values $<0.05$ were considered as statistically significant.) 


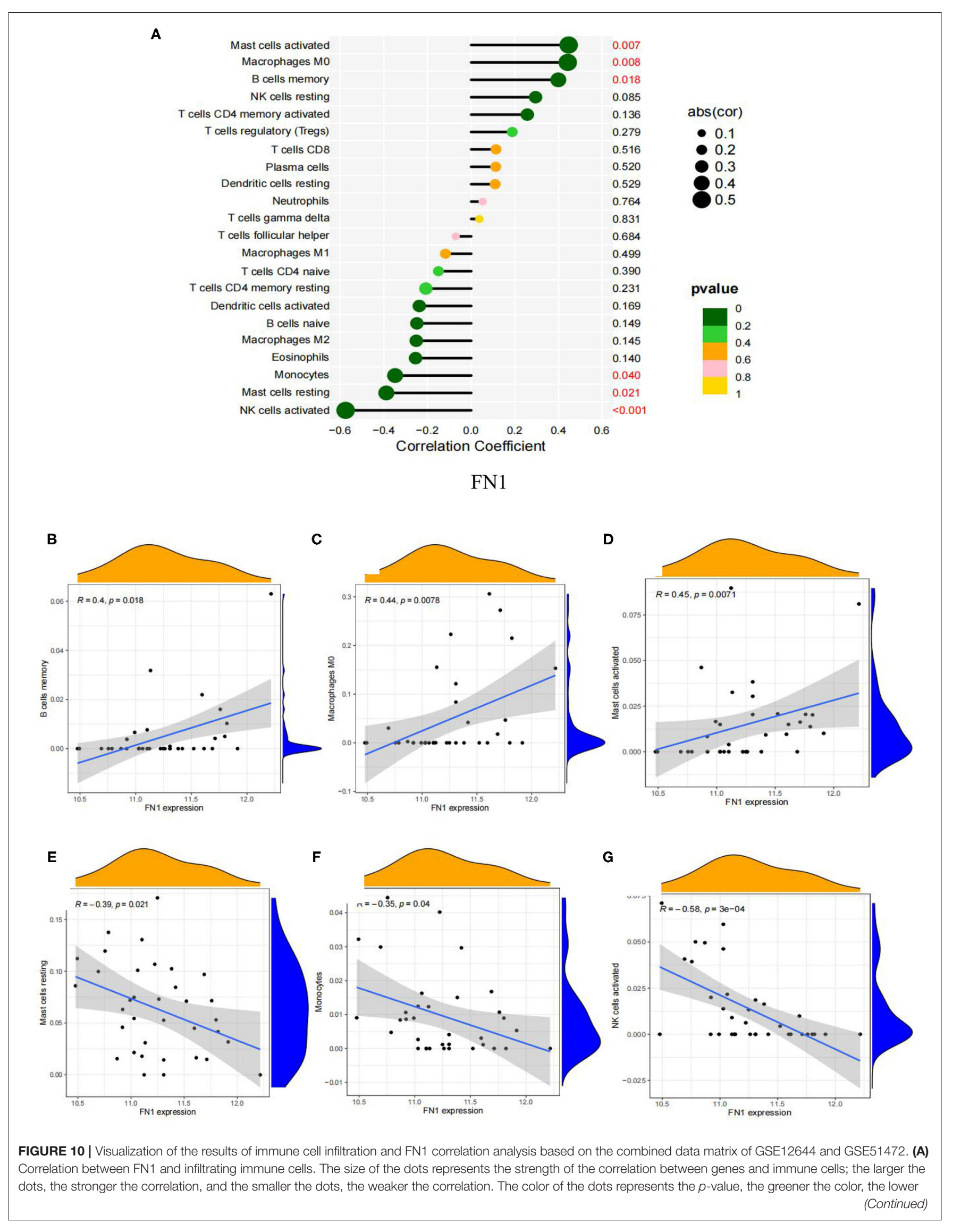


FIGURE 10 | the $p$-value, and the yellower the color, the larger the $p$-value $<0.05$ was considered statistically significant. (B) The correlation analysis in the expression of FN1 and B cells memory. (C) The correlation analysis in the expression of FN1 and Macrophages MO. (D) The correlation analysis in the expression of FN1 and Mast cells activated. (E) Correlation between FN1 and Mast cells resting. (F) The correlation analysis in the expression of FN1 and Monocytes. (G) The correlation analysis in the expression of FN1 and NK cells activated.

TABLE 2 | List of the 10 most significant small molecule drugs that can reverse the calcified status of AVC.

\begin{tabular}{llll}
\hline AVC tissue & CMap name & Enrichment & $P$-value \\
\hline & Yohimbic acid & -0.899 & 0.00192 \\
& PF-00539745-00 & -0.769 & 0.0252 \\
HVemicholinium & -0.665 & 0.02847 \\
& Brinzolamide & -0.655 & 0.03284 \\
& Pepstatin & -0.645 & 0.0377 \\
& Lithocholic acid & -0.612 & 0.01114 \\
& Minoxidil & -0.567 & 0.04652 \\
& Salbutamol & 0.625 & 0.02159 \\
& Colchicine & 0.634 & 0.00735 \\
& Physostigmine & 0.636 & 0.04307
\end{tabular}

AVC, aortic valve calcification.

tissues, and naïve B cells, plasma cells, activated natural killer cells, and monocytes are significantly expressed in normal tissues. In addition, FN1 was significantly expressed in the AVC tissue. Correlation analysis showed that memory B cells, macrophages M0, activated mast cells were positively correlated with FN1, and resting mast cells, monocytes, as well as activated natural killer cells were negatively correlated with FN1, which meaning that the high expression of FN1 tissues greatly promotes the infiltration of memory B cells, macrophages M0 to the tissues, on the contrary, may inhibit the activation of activated natural killer cells and monocytes. Therefore, FN1 and a variety of inflammatory cells participate in the progress of AVC, which provides effective evidence for further research on the molecular mechanism of AVC in the future. Inflammatory and immune circulating cells, such as memory B cells, neutrophils and macrophages, have been shown to have a significant influence in the development of heart-related diseases in the prior (69), which is consistent with our research. Endothelial damage caused by increased mechanical stress and decreased shear stress is thought to be the first stage in AVC development (70). As a consequence, localized subendothelial plaque-like lesions may be detected, involving infiltration of inflammatory cells, buildup of subendothelial lipids, breakdown of the extracellular matrix, and fracturing of the surrounding elastic lamina (71). Furthermore, oxidized lipids also induce an inflammatory response in valve tissue. In response to the uptake of oxidized lipids, macrophages, monocytes, CD4+ and CD8+ T lymphocytes, and mast cells in the surrounding area become activated (10). Monocytes and macrophages stimulate the osteogenic differentiation of valvular interstitial cells and calcification via secretion of tumor necrosis factor, which is followed by activation of NF- $\kappa \mathrm{B}$ and IL- $1 \beta$ and IL6 (72). Following this, as a result of endothelial dysfunction and inflammation, localized cell death leads to the release of apoptotic bodies, which promote microcalcification. This process is further promoted by the release of extracellular vesicles by macrophages and valvular interstitial cells $(73,74)$. Such calcification induces an even stronger immune response, resulting in a vicious loop and ultimately leading to the disease's propagation phase. AVC patients' natural killer cells accumulate in cardiac valves and blood circulation, which has been associated to an increase in their valve pressure gradient, according to Mazur's research (75). We suggest that the enormous amount of evidence described earlier, combined with our present study findings, indicates that a variety of infiltrating immune cells play an essential role in AVC and future study should be focused on this point.

The CMAP database, which contains 7,056 gene expression profiles triggered by 1,309 small compounds, is commonly used to investigate the unknown functions of currently available medications in disease (76). To begin, the 337 DEGs were categorized into up- and down-regulated categories. Then, these genes from two groups were uploaded to the CMAP database to identify possible small therapeutic compounds, with a cutoff value of $P<0.05$ used. Finally, enrichment scores $(-1$ to +1 ) were generated to determine the similarity between genes and medicines. Specifically, an enrichment score $>0$ indicated that the molecules had potential synergistic effects with AVC, implying that they could mimic the biological state of the cells that induce AVC; whereas, an enrichment score $<0$ indicated that the molecules had potential inhibitory action with AVC, implying that they could reverse the AVC state and serve as therapeutic agents. We therefore successfully identified the top 10 small molecules with the smallest enrichment scores, of which yohimbic acid was the first. However, very little research has been done on yohimbic acid, with only a few studies reporting its vasodilatory (77) and sympathetic activity effects (78). There is still a lack of a large pharmacological base to verify its function. Therefore, further studies are necessary to confirm its reversal or delaying effect on AVC in the future.

This study has several limitations. First, it was retrospective in nature; thus, there was a lack of critical clinical information. Secondly, the GSE83453 validation cohort included a small number of cases, which could be considered as a constraint. Thirdly, it is challenging to account for critical aspects such as area, race, and age. Considering that AVC is caused by a multitude of environmental and genetic variables, certain unmeasurable factors, such as geography and family history, need additional investigation. Additionally, potential key genes should be validated in clinical samples using RT-qPCR. However, since there are not enough normal aortic valve samples in our department, it is difficult to carry out the verification experiment. In the future, we will collect the aortic valve tissue transplanted in our department to further explore the mechanism of FN1 on AVC. Finally, the method by which these genes operate is unknown. Additional evidence is evaluated to confirm the biological basis. 
In conclusion, we present bioinformatic evidence demonstrating that FN1 might be a potential biomarker for discrimination of AVC. Yohimbic acid was also identified as a potential anti-calcification drug in calcified aortic disease $(P$ $<0.05)$. Given that the pathogenic processes of AVC remain unknown, our findings may have a wide influence on AVC biology and treatment. Nonetheless, larger sample sizes and further mechanistic investigations are required to corroborate our conclusions.

\section{DATA AVAILABILITY STATEMENT}

The datasets presented in this study can be found in online repositories. The names of the repository/repositories and accession number(s) can be found in the article/Supplementary Material.

\section{AUTHOR CONTRIBUTIONS}

TX was engaged for conception and writing-original draft production. $\mathrm{XZ}$ and $\mathrm{SH}$ for methodology. LP, T-CZ, TF, and Y-HD for sofware. Y-XL for writing-review and editing. All

\section{REFERENCES}

1. Iung B, Vahanian A. Epidemiology of valvular heart disease in the adult. Nat Rev Cardiol. (2011) 8:162-72. doi: 10.1038/nrcardio.2010.202

2. Liu M, Luo M, Sun H, Ni B, Shao Y. Integrated bioinformatics analysis predicts the key genes involved in aortic valve calcification: from hemodynamic changes to extracellular remodeling. Tohoku J Exp Med. (2017) 243:26373. doi: $10.1620 /$ tjem. 243.263

3. Vahanian A, Alfieri O, Andreotti F, Antunes MJ, Baron-Esquivias G, Baumgartner $\mathrm{H}$, et al. Guidelines on the management of valvular heart disease (version 2012): the Joint Task Force on the Management of Valvular Heart Disease of the European Society of Cardiology (ESC) and the European Association for Cardio-Thoracic Surgery (EACTS). Eur J Cardiothorac Surg. (2012) 42:S1-44. doi: 10.1093/eurheartj/ehs109

4. Lindman BR, Clavel MA, Mathieu P, Iung B, Lancellotti P, Otto $\mathrm{CM}$, et al. Calcific aortic stenosis. Nat Rev Dis Primers. (2016) 2:16006. doi: 10.1038/nrdp.2016.6

5. Bull S, Loudon M, Francis JM, Joseph J, Gerry S, Karamitsos TD, et al. A prospective, double-blind, randomized controlled trial of the angiotensinconverting enzyme inhibitor Ramipril In Aortic Stenosis (RIAS trial). Eur Heart J Cardiovasc Imaging. (2015) 16:834-41. doi: 10.1093/ehjci/ jev043

6. Rossebo AB, Pedersen TR, Boman K, Brudi P, Chambers JB, Egstrup K, et al. Intensive lipid lowering with simvastatin and ezetimibe in aortic stenosis. $N$ Engl J Med. (2008) 359:1343-56. doi: 10.1056/NEJMoa0804602

7. Cowell SJ, Newby DE, Prescott RJ, Bloomfield P, Reid J, Northridge DB, et al. A randomized trial of intensive lipid-lowering therapy in calcific aortic stenosis. N Engl J Med. (2005) 352:2389-97. doi: 10.1056/NEJMoa043876

8. Chan KL, Teo K, Dumesnil JG, Ni A, Tam J, Investigators A. effect of lipid lowering with rosuvastatin on progression of aortic stenosis: results of the aortic stenosis progression observation: measuring effects of rosuvastatin (ASTRONOMER) trial. Circulation. (2010) 121:306-14. doi: 10.1161/CIRCULATIONAHA.109.900027

9. Kang DH, Park SJ, Rim JH, Yun SC, Kim DH, Song JM, et al. Early surgery versus conventional treatment in asymptomatic very severe aortic stenosis. Circulation. (2010) 121:15029. doi: 10.1161/CIRCULATIONAHA.109.909903 authors have reviewed and approved the published version of the work.

\section{FUNDING}

This study was sponsored by funds from the Chinese National Natural Science Foundation (No. 82060093), the Science and Technology Department of Yunnan Province (No. 202101AY070001-211 and No. 2019FE001-268), and Yunnan Province's Key Laboratory of Cardiovascular Disease (No. 2018DG008).

\section{ACKNOWLEDGMENTS}

GEO facilitated in the completion of this work. We would like to express our gratitude to the GEO network for freely sharing huge amounts of data.

\section{SUPPLEMENTARY MATERIAL}

The Supplementary Material for this article can be found online at: https://www.frontiersin.org/articles/10.3389/fcvm. 2022.832591/full\#supplementary-material

10. Otto CM, Kuusisto J, Reichenbach DD, Gown AM, O'Brien KD. Characterization of the early lesion of "degenerative" valvular aortic stenosis. Histological and immunohistochemical studies. Circulation. (1994) 90:844-53. doi: 10.1161/01.CIR.90.2.844

11. Mohler ER, 3rd, Gannon F, Reynolds C, Zimmerman R, Keane MG, Kaplan FS. Bone formation and inflammation in cardiac valves. Circulation. (2001) 103:1522-8. doi: 10.1161/01.CIR.103.11.1522

12. O'Brien KD, Shavelle DM, Caulfield MT, McDonald TO, Olin-Lewis K, Otto $\mathrm{CM}$, et al. Association of angiotensin-converting enzyme with low-density lipoprotein in aortic valvular lesions and in human plasma. Circulation. (2002) 106:2224-30. doi: 10.1161/01.CIR.0000035655.45453.D2

13. Jian B, Narula N, Li QY, Mohler ER, 3rd, Levy RJ. Progression of aortic valve stenosis: TGF-betal is present in calcified aortic valve cusps and promotes aortic valve interstitial cell calcification via apoptosis. Ann Thorac Surg. (2003) 75:457-65; discussion 65-6. doi: 10.1016/S0003-4975(02)04312-6

14. Kaden JJ, Kilic R, Sarikoc A, Hagl S, Lang S, Hoffmann U, et al. Tumor necrosis factor alpha promotes an osteoblast-like phenotype in human aortic valve myofibroblasts: a potential regulatory mechanism of valvular calcification. Int J Mol Med. (2005) 16:869-72. doi: 10.3892/ijmm.16.5.869

15. Yutzey KE, Demer LL, Body SC, Huggins GS, Towler DA, Giachelli CM, et al. Calcific aortic valve disease: a consensus summary from the Alliance of Investigators on Calcific Aortic Valve Disease. Arterioscler Thromb Vasc Biol. (2014) 34:2387-93. doi: 10.1161/ATVBAHA.114.302523

16. Bosse Y, Miqdad A, Fournier D, Pepin A, Pibarot P, Mathieu P. Refining molecular pathways leading to calcific aortic valve stenosis by studying gene expression profile of normal and calcified stenotic human aortic valves. Circ Cardiovasc Genet. (2009) 2:489-98. doi: 10.1161/CIRCGENETICS.108.8 20795

17. Ohukainen P, Syvaranta S, Napankangas J, Rajamaki K, Taskinen P, Peltonen T, et al. MicroRNA-125b and chemokine CCL4 expression are associated with calcific aortic valve disease. Ann Med. (2015) 47:4239. doi: 10.3109/07853890.2015.1059955

18. Barrett T, Wilhite SE, Ledoux P, Evangelista C, Kim IF, Tomashevsky M, et al. NCBI GEO: archive for functional genomics data sets-update. Nucleic Acids Res. (2013) 41:D991-5. doi: 10.1093/nar/gks1193

19. Chen C, Grennan K, Badner J, Zhang D, Gershon E, Jin L, et al. Removing batch effects in analysis of expression microarray data: 
an evaluation of six batch adjustment methods. PLoS ONE. (2011) 6:e17238. doi: 10.1371/journal.pone.0017238

20. Guauque-Olarte S, Droit A, Tremblay-Marchand J, Gaudreault N, Kalavrouziotis D, Dagenais F, et al. RNA expression profile of calcified bicuspid, tricuspid, and normal human aortic valves by RNA sequencing. Physiol Genomics. (2016) 48:74961. doi: 10.1152/physiolgenomics.00041.2016

21. Ritchie ME, Phipson B, Wu D, Hu Y, Law CW, Shi W, et al. limma powers differential expression analyses for RNA-sequencing and microarray studies. Nucleic Acids Res. (2015) 43:e47. doi: 10.1093/nar/gkv007

22. Ito K, Murphy D. Application of ggplot2 to pharmacometric graphics. CPT Pharmacometrics Syst Pharmacol. (2013) 2:e79. doi: 10.1038/psp.2013.56

23. Yu G, Wang LG, Han Y, He QY. clusterProfiler: an R package for comparing biological themes among gene clusters. OMICS. (2012) 16:2847. doi: 10.1089/omi.2011.0118

24. Szklarczyk D, Morris JH, Cook H, Kuhn M, Wyder S, Simonovic M, et al. The STRING database in 2017: quality-controlled protein-protein association networks, made broadly accessible. Nucleic Acids Res. (2017) 45:D3628. doi: 10.1093/nar/gkw937

25. Chin $\mathrm{CH}$, Chen $\mathrm{SH}, \mathrm{Wu} \mathrm{HH}, \mathrm{Ho} \mathrm{CW}$, Ko MT, Lin CY. cytoHubba: identifying hub objects and sub-networks from complex interactome. BMC Syst Biol. (2014) 8(Suppl 4):S11. doi: 10.1186/1752-0509-8-S4-S11

26. Tibshirani R. Regression shrinkage and selection via the lasso. J R Stat Soc. (1996) 58:267-88. doi: 10.1111/j.2517-6161.1996.tb02080.x

27. Huang S, Cai N, Pacheco PP, Narrandes S, Wang Y, Xu W. Applications of support vector machine (SVM) learning in cancer genomics. Cancer Genom Proteomics. (2018) 15:41-51. doi: 10.21873/cgp.20063

28. Friedman J, Hastie T, Tibshirani R. Regularization paths for generalized linear models via coordinate descent. J Stat Softw. (2010) 33:1-22. doi: $10.18637 /$ jss.v033.i01

29. Huang ML, Hung YH, Lee WM, Li RK, Jiang BR. SVM-RFE based feature selection and Taguchi parameters optimization for multiclass SVM classifier. Sci World J. (2014) 2014:795624. doi: 10.1155/2014/795624

30. Friendly M. Corrgrams: Exploratory displays for correlation matrices. Am Stat. (2002) 56:316-24. doi: 10.1198/000313002533

31. Freeman RV, Otto CM. Spectrum of calcific aortic valve disease: pathogenesis, disease progression, and treatment strategies. Circulation. (2005) 111:331626. doi: 10.1161/CIRCULATIONAHA.104.486738

32. Coffey S, Cox B, Williams MJ. The prevalence, incidence, progression, and risks of aortic valve sclerosis: a systematic review and meta-analysis. J Am Coll Cardiol. (2014) 63:2852-61. doi: 10.1016/j.jacc.2014.04.018

33. Mathieu P, Boulanger MC. Basic mechanisms of calcific aortic valve disease. Can J Cardiol. (2014) 30:982-93. doi: 10.1016/j.cjca.2014.03.029

34. Kostyunin AE, Yuzhalin AE, Ovcharenko EA, Kutikhin AG. Development of calcific aortic valve disease: do we know enough for new clinical trials? J Mol Cell Cardiol. (2019) 132:189-209. doi: 10.1016/j.yjmcc.2019.05.016

35. Dutta P, Lincoln J. Calcific aortic valve disease: a developmental biology perspective. Curr Cardiol Rep. (2018) 20:21. doi: 10.1007/s1 1886-018-0968-9

36. Gould ST, Srigunapalan S, Simmons CA, Anseth KS. Hemodynamic and cellular response feedback in calcific aortic valve disease. Circ Res. (2013) 113:186-97. doi: 10.1161/CIRCRESAHA.112.300154

37. Lindman BR, Bonow RO, Otto CM. Current management of calcific aortic stenosis. Circ Res. (2013) 113:223-37. doi: 10.1161/CIRCRESAHA.111.300084

38. Zhao Y, Xia Z, Lin T, Yin Y. Significance of hub genes and immune cell infiltration identified by bioinformatics analysis in pelvic organ prolapse. PeerJ. (2020) 8:e9773. doi: 10.7717/peerj.9773

39. Wang D, Xiong T, Yu W, Liu B, Wang J, Xiao K, et al. Predicting the key genes involved in aortic valve calcification through integrated bioinformatics analysis. Front Genet. (2021) 12:650213. doi: 10.3389/fgene.2021.650213

40. Zhang Y, Ma L. Identification of key genes and pathways in calcific aortic valve disease by bioinformatics analysis. J Thorac Dis. (2019) 11:541726. doi: $10.21037 /$ jtd.2019.11.57

41. Yu R, Zhang J, Zhuo Y, Hong X, Ye J, Tang S, et al. Identification of diagnostic signatures and immune cell infiltration characteristics in rheumatoid arthritis by integrating bioinformatic analysis and machine-learning strategies. Front Immunol. (2021) 12:724934. doi: 10.3389/fimmu.2021.724934

42. Pirooznia M, Yang JY, Yang MQ, Deng Y. A comparative study of different machine learning methods on microarray gene expression data. BMC Genomics. (2008) 9(Suppl 1):S13. doi: 10.1186/1471-2164-9S1-S13

43. Akter S, Xu D, Nagel SC, Bromfield JJ, Pelch K, Wilshire GB, et al. Machine learning classifiers for endometriosis using transcriptomics and methylomics data. Front Genet. (2019) 10:766. doi: 10.3389/fgene.2019.00766

44. Cote N, Mahmut A, Bosse Y, Couture C, Page S, Trahan S, et al. Inflammation is associated with the remodeling of calcific aortic valve disease. Inflammation. (2013) 36:573-81. doi: 10.1007/s10753-012-9579-6

45. Lacalle RA, Blanco R, Carmona-Rodriguez L, Martin-Leal A, Mira E, Manes S. Chemokine receptor signaling and the hallmarks of cancer. Int Rev Cell Mol Biol. (2017) 331:181-244. doi: 10.1016/bs.ircmb.2016.09.011

46. Dweck MR, Khaw HJ, Sng GK, Luo EL, Baird A, Williams MC, et al. Aortic stenosis, atherosclerosis, and skeletal bone: is there a common link with calcification and inflammation? Eur Heart J. (2013) 34:156774. doi: 10.1093/eurheartj/eht034

47. Yip CY, Simmons CA. The aortic valve microenvironment and its role in calcific aortic valve disease. Cardiovasc Pathol. (2011) 20:17782. doi: 10.1016/j.carpath.2010.12.001

48. Mahler GJ, Butcher JT. Inflammatory regulation of valvular remodeling: the $\operatorname{good}(?)$, the bad, and the ugly. Int J Inflam. (2011) 2011:721419. doi: 10.4061/2011/721419

49. Tintut Y, Patel J, Parhami F, Demer LL. Tumor necrosis factor-alpha promotes in vitro calcification of vascular cells via the cAMP pathway. Circulation. (2000) 102:2636-42. doi: 10.1161/01.CIR.102.21.2636

50. Olsson M, Dalsgaard CJ, Haegerstrand A, Rosenqvist M, Ryden L, Nilsson J. Accumulation of T lymphocytes and expression of interleukin-2 receptors in nonrheumatic stenotic aortic valves. J Am Coll Cardiol. (1994) 23:116270. doi: 10.1016/0735-1097(94)90606-8

51. Venardos N, Nadlonek NA, Zhan Q, Weyant MJ, Reece TB, Meng X, et al. Aortic valve calcification is mediated by a differential response of aortic valve interstitial cells to inflammation. J Surg Res. (2014) 190:18. doi: $10.1016 /$ j.jss.2013.11.373

52. Yao Y, Watson AD, Ji S, Bostrom KI. Heat shock protein 70 enhances vascular bone morphogenetic protein-4 signaling by binding matrix Gla protein. Circ Res. (2009) 105:575-84. doi: 10.1161/CIRCRESAHA.109.202333

53. Hjortnaes J, New SE, Aikawa E. Visualizing novel concepts of cardiovascular calcification. Trends Cardiovasc Med. (2013) 23:71-9. doi: 10.1016/j.tcm.2012.09.003

54. Maurer LM, Ma W, Mosher DF. Dynamic structure of plasma fibronectin. Crit Rev Biochem Mol Biol. (2015) 51:213-27. doi: 10.1080/10409238.2016.1184224

55. Schwarzbauer JE, DeSimone DW. Fibronectins, their fibrillogenesis, and in vivo functions. Cold Spring Harb Perspect Biol. (2011) 3:a005041. doi: 10.1101/cshperspect.a005041

56. Gao W, Liu Y, Qin R, Liu D, Feng Q. Silence of fibronectin 1 increases cisplatin sensitivity of non-small cell lung cancer cell line. Biochem Biophys Res Commun. (2016) 476:35-41. doi: 10.1016/j.bbrc.2016.05.081

57. Kimura E, Kanzaki T, Tahara K, Hayashi H, Hashimoto S, Suzuki A, et al. Identification of citrullinated cellular fibronectin in synovial fluid from patients with rheumatoid arthritis. Mod Rheumatol. (2014) 24:7669. doi: 10.3109/14397595.2013.879413

58. Doddapattar P, Gandhi C, Prakash P, Dhanesha N, Grumbach IM, Dailey ME, et al. Fibronectin splicing variants containing extra domain a promote atherosclerosis in mice through toll-like receptor 4. Arterioscler Thromb Vasc Biol. (2015) 35:2391-400. doi: 10.1161/ATVBAHA.115. 306474

59. Sens C, Huck K, Pettera S, Uebel S, Wabnitz G, Moser M, et al. Fibronectins containing extradomain A or B enhance osteoblast differentiation via distinct integrins. J Biol Chem. (2017) 292:7745-60. doi: 10.1074/jbc.M116.739987

60. Yang C, Wang C, Zhou J, Liang Q, He F, Li F, et al. Fibronectin 1 activates WNT/beta-catenin signaling to induce osteogenic differentiation via integrin betal interaction. Lab Invest. (2020) 100:1494-502. doi: 10.1038/s41374-020-0451-2

61. Bostrom KI, Rajamannan NM, Towler DA. The regulation of valvular and vascular sclerosis by osteogenic morphogens. Circ Res. (2011) 109:56477. doi: 10.1161/CIRCRESAHA.110.234278

62. Jun HK, Jung YJ, Choi BK. Inflammasome activators induce fibronectin expression and release in macrophages. Cell Microbiol. (2017) 19. doi: $10.1111 / \mathrm{cmi} .12695$ 
63. Lemanska-Perek A, Krzyzanowska-Golab D, Pupek M, Klimeczek P, Witkiewicz W, Katnik-Prastowska I. Analysis of soluble molecular fibronectin-fibrin complexes and EDA-fibronectin concentration in plasma of patients with atherosclerosis. Inflammation. (2016) 39:1059-68. doi: 10.1007/s10753-016-0336-0

64. Kim DH, Yoo TH, Lee SH, Kang HY, Nam BY, Kwak SJ, et al. Gamma linolenic acid exerts anti-inflammatory and antifibrotic effects in diabetic nephropathy. Yonsei Med J. (2012) 53:1165-75. doi: 10.3349/ymj.2012.53.6.1165

65. Clemmensen I, Holund B, Andersen RB. Fibrin and fibronectin in rheumatoid synovial membrane and rheumatoid synovial fluid. Arthritis Rheum. (1983) 26:479-85. doi: 10.1002/art.1780260405

66. Xie DL, Meyers R, Homandberg GA. Fibronectin fragments in osteoarthritic synovial fluid. J Rheumatol. (1992) 19:1448-52.

67. Man SF, Xing L, Connett JE, Anthonisen NR, Wise RA, Tashkin DP, et al. Circulating fibronectin to C-reactive protein ratio and mortality: a biomarker in COPD? Eur Respir J. (2008) 32:1451-7. doi: 10.1183/09031936.00153207

68. Vavalle JP, Wu SS, Hughey R, Madamanchi NR, Stouffer GA. Plasma fibronectin levels and coronary artery disease. J Thromb Haemost. (2007) 5:864-6. doi: 10.1111/j.1538-7836.2007.02420.x

69. Falk E. Pathogenesis of atherosclerosis. J Am Coll Cardiol. (2006) 47(8 Suppl):C7-12. doi: 10.1016/j.jacc.2005.09.068

70. Butcher JT, Nerem RM. Valvular endothelial cells and the mechanoregulation of valvular pathology. Philos Trans R Soc Lond B Biol Sci. (2007) 362:144557. doi: 10.1098/rstb.2007.2127

71. O’Brien KD, Reichenbach DD, Marcovina SM, Kuusisto J, Alpers CE, Otto CM. Apolipoproteins B, (a), and E accumulate in the morphologically early lesion of "degenerative" valvular aortic stenosis. Arterioscler Thromb Vasc Biol. (1996) 16:523-32. doi: 10.1161/01.ATV.16.4.523

72. Galeone A, Brunetti G, Oranger A, Greco G, Di Benedetto A, Mori G, et al. Aortic valvular interstitial cells apoptosis and calcification are mediated by TNF-related apoptosis-inducing ligand. Int J Cardiol. (2013) 169:296304. doi: 10.1016/j.ijcard.2013.09.012

73. New SE, Goettsch C, Aikawa M, Marchini JF, Shibasaki M, Yabusaki K, et al. Macrophage-derived matrix vesicles: an alternative novel mechanism for microcalcification in atherosclerotic plaques. Circ Res. (2013) 113:727. doi: 10.1161/CIRCRESAHA.113.301036
74. Proudfoot D, Skepper JN, Hegyi L, Bennett MR, Shanahan CM, Weissberg PL. Apoptosis regulates human vascular calcification in vitro: evidence for initiation of vascular calcification by apoptotic bodies. Circ Res. (2000) 87:1055-62. doi: 10.1161/01.RES.87. 11.1055

75. Mazur P, Mielimonka A, Natorska J, Wypasek E, Gaweda B, Sobczyk D, et al. Lymphocyte and monocyte subpopulations in severe aortic stenosis at the time of surgical intervention. Cardiovasc Pathol. (2018) 35:17. doi: 10.1016/j.carpath.2018.03.004

76. Subramanian A, Narayan R, Corsello SM, Peck DD, Natoli TE, Lu X, et al. A next generation connectivity map: L1000 platform and the first 1,000,000 profiles. Cell. (2017) 171:1437-52 e17. doi: 10.1016/j.cell.2017. 10.049

77. Raymond H. Demonstration of the direct vasodilatory action of yohimbic acid and Py-tetrahydroquinoline. C R Hebd Seances Acad Sci. (1960) 250:4473-5.

78. Raymond H. Sympatholytic activity and toxicity of an amphoteric demethylated derivative of yohimbine: yohimbic acid. C R Hebd Seances Acad Sci. (1951) 233:1069-71.

Conflict of Interest: The authors declare that the research was conducted in the absence of any commercial or financial relationships that could be construed as a potential conflict of interest.

Publisher's Note: All claims expressed in this article are solely those of the authors and do not necessarily represent those of their affiliated organizations, or those of the publisher, the editors and the reviewers. Any product that may be evaluated in this article, or claim that may be made by its manufacturer, is not guaranteed or endorsed by the publisher.

Copyright (C) 2022 Xiong, Han, Pu, Zhang, Zhan, Fu, Dai and Li. This is an openaccess article distributed under the terms of the Creative Commons Attribution License (CC BY). The use, distribution or reproduction in other forums is permitted, provided the original author(s) and the copyright owner(s) are credited and that the original publication in this journal is cited, in accordance with accepted academic practice. No use, distribution or reproduction is permitted which does not comply with these terms. 